\title{
Extended thermodynamics and complexity in gravitational Chern-Simons theory
}

\author{
Antonia M. Frassino, ${ }^{a, b}$ Robert B. Mann ${ }^{c, d}$ and Jonas R. Mureika ${ }^{e}$ \\ ${ }^{a}$ Departament de Física Quàntica i Astrofísica, Institut de Ciències del Cosmos, \\ Universitat de Barcelona, \\ Martí i Franquès 1, E-08028 Barcelona, Spain \\ ${ }^{b}$ Département de Physique Théorique and Center for Astroparticle Physics, Université de Genève, \\ 24 quai Ansermet, $\mathrm{CH}-1211$ Genève 4, Switzerland \\ ${ }^{c}$ Perimeter Institute for Theoretical Physics, \\ 31 Caroline St. N., Waterloo, Ontario N2L 2Y5, Canada \\ ${ }^{d}$ Department of Physics and Astronomy, University of Waterloo, \\ Waterloo, Ontario N2L 3G1, Canada \\ ${ }^{e}$ Department of Physics, Loyola Marymount University, \\ Los Angeles, California, 90045, U.S.A. \\ E-mail: antoniam.frassino@icc.ub.edu, rbmann@uwaterloo.ca, \\ jmureika@lmu.edu
}

ABSTRACT: We study several aspects of the extended thermodynamics of BTZ black holes with thermodynamic mass $M=\alpha m+\gamma \frac{j}{\ell}$ and angular momentum $J=\alpha j+\gamma \ell m$, for general values of the parameters $(\alpha, \gamma)$ ranging from regular $(\alpha=1, \gamma=0)$ to exotic $(\alpha=0, \gamma=1)$. We show that there exist two distinct behaviours for the black holes, one when $\alpha>\gamma$ ("mostly regular"), and the other when $\gamma<\alpha$ ("mostly exotic"). We find that the Smarr formula holds for all $(\alpha, \gamma)$. We derive the corresponding thermodynamic volumes, which we find to be positive provided $\alpha$ and $\gamma$ satisfy a certain constraint. The dependence of pressure on volume is unremarkable and strictly decreasing when $\alpha>\gamma$, but a maximum volume emerges for large $J \gg T$ when $\gamma>\alpha$; consequently an exotic black hole of a given horizon circumference and temperature can exist in two distinct anti de Sitter backgrounds. We compute the reverse isoperimetric ratio, and study the Gibbs free energy and criticality conditions for each. Finally we investigate the complexity growth of these objects and find that they are all proportional to the complexity of the BTZ black hole. Somewhat surprisingly, purely exotic BTZ black holes have vanishing complexity growth.

KEYworDS: Black Holes, AdS-CFT Correspondence, Models of Quantum Gravity

ArXiv EPrint: 1906.07190 


\section{Contents}

1 Introduction 1

2 Black hole solution from gravitational Chern-Simons 3

2.1 Thermodynamics 6

$\begin{array}{ll}2.2 & \text { Extended phase space }\end{array}$

3 Pressure-volume behaviour $\quad 9$

4 Reverse isoperimetric ratio $\quad 12$

5 Gibbs free energy and critical behaviour $\quad \mathbf{1 2}$

$\begin{array}{lll}5.1 & \text { Free energy with fixed- } \Omega & 15\end{array}$

6 Complexity growth rate $\quad 16$

$\begin{array}{ll}\text { 6.1 Complexity of the exotic BTZ black hole } & 17\end{array}$

$\begin{array}{lll}7 & \text { Conclusions } & 19\end{array}$

A BTZ black hole complexity growth $\quad 20$

A.1 General definition of the WdW patch 20

A.2 Rotating BTZ in Einstein-Cartan formalism 21

A.3 Volume contributions to the WdW patch 23

A.4 Joint contributions 24

$\begin{array}{lll}\text { A.5 Boundary counterterm for rotating BTZ } & 25\end{array}$

$\begin{array}{ll}\text { B Alternate possibilities } & 28\end{array}$

\section{Introduction}

Black hole physics and thermodynamics have an interesting relationship reaching back over more than four decades [1-3], one that remains an active research area of considerable import in theoretical physics, primarily due to its implications for quantum gravity. It is particularly relevant in spacetimes with a negative cosmological constant as it provides connections between otherwise distinct theoretical concepts in the context of the AdS/CFT correspondence conjecture [4], which relates string theories (and therefore gravitation) formulated on asymptotically-anti de Sitter (AdS) spacetimes to a conformal field theory on the spacetime boundary.

The cosmological constant, $\Lambda$, has generally been regarded as an inert parameter in black hole thermodynamics, one that sets a new length scale but does little else in this 
context. In recent years, however, the role of $\Lambda$ has been found to be quite significant, and a new sub-discipline called "black hole chemistry" has emerged [5]. In this approach, each thermodynamic parameter in an asymptotically AdS black hole has a chemical counterpart in representations of the first law, with $\Lambda$ associated with pressure. From a cosmological perspective a negative cosmological constant induces a vacuum pressure, and so the former association is quite natural. A new $P V$ term is thereby introduced into the first law, where $V$ is the volume conjugate to $P$. The presence of a black hole in the spacetime can be regarded as a displacement of vacuum energy, leading to an interpretation of the black hole mass $M$ as a gravitational analogue of chemical enthalpy $[5,6]$.

Subsequent investigation has indicated that black hole thermodynamics has a very close correspondence with real world systems [6-9] including Van der Waals fluids [10], reentrant phase transitions [11-13], triple points analogous to the triple point in water [14] and nuclear matter systems [15]. More recently a holographic interpretation of some of these phenomena has been studied [16-18], and it has been shown that black holes can be regarded as holographic heat engines, where renormalization group flow performs the cycles [19-22]. Very recently a class of hairy black holes was discovered that exhibit superfluid phase transitions analogous to those of superfluid helium [23]. A thorough review describing these phenomena and much more has recently appeared [24].

One of the first insights of black hole chemistry is that in order to satisfy the Smarr relation [25], it is necessary to treat $\Lambda$ as thermodynamic pressure. In any dimension the first law and its associated Smarr relation are

$$
\begin{aligned}
d\left(G_{D} M\right) & =T d S+\Omega d J+V d P \\
(D-3) G_{D} M & =(D-2) T S+(D-2) \Omega J-2 V P+(D-3) \Phi Q
\end{aligned}
$$

for a charged singly-rotating black hole, where $J$ is its angular momentum, $\Omega$ its angular velocity, $T$ its temperature and $S$ its entropy, and the $D$-dimensional Newton constant $G_{D}$ has been explicitly retained. The thermodynamic pressure $P$ is

$$
P=-\frac{\Lambda}{8 \pi}=\frac{(D-2)(D-1)}{16 \pi l^{2}}
$$

and its conjugate volume is $V$. Despite factors of $(D-3)$ and $(D-2)$ appearing in the above formulae, it has recently been shown that (1.2) holds in both the $D \rightarrow 3$ and $D \rightarrow 2$ limits [26]. Although there were no phase transitions in either dimension, the $D=3$ case led to a number of interesting findings.

In particular, the definition of thermodynamic volume was somewhat subtle, with the volume depending on both horizon size and charge, quite unlike the situation for charged black holes in higher dimensions $[10,12]$. Conversely, retaining a definition of volume depending only on horizon size necessitates introduction of a new work term associated with the renormalization scale of the black hole mass, in turn modifying both the Smarr formula (1.2) and first law (1.1) in $D=3$.

Our interest in this paper is in better understanding of the $D=3$ case in the presence of a gravitational Chern-Simons term in the three dimensional action. We consequently can discuss features of the so-called "exotic" black hole solution. These are black holes 
whose metric is of the same form as the $D=3 \mathrm{BTZ}$ black hole but whose parameters of mass and charge are interchanged. These solutions were first obtained by considering the behaviour of topological matter in $D=3$ gravity [27]. Such matter does not couple to the spacetime metric but rather only to the connection. The net effect is that the (negative) cosmological constant becomes a constant of integration associated with the topological matter, and the conserved charges of mass and angular momentum become interchanged with one another. Even stranger was that the entropy of these black holes is proportional to the area of their inner horizons [27]. This situation can occur in other theories of gravity in $D=3$ [28], and it was recently shown that the entropy of such exotic black holes still has a statistical interpretation [29].

In this paper we consider the chemistry of these exotic black holes. We are particularly interested in understanding the generalized first law (1.1) and Smarr formula (1.2) for these objects, along with their thermodynamic volume and its properties. We find a number of interesting distinctions between these two cases. The Gibbs free energy is in general always larger for exotic black holes than for their standard BTZ counterparts. Furthermore, we find that in certain regions of parameter space an exotic black hole can have two distinct pressures for the same thermodynamic volume. Since volume is proportional to radius, this means that an exotic black hole of a given horizon circumference and temperature can exist in two distinct AdS backgrounds. These black holes have a maximum size at a given temperature. Finally, we find that the volume of a sufficiently small and sufficiently exotic black hole violates the Reverse Isoperimetric Inequality [30]; only for $\alpha \geq \gamma$ is this inequality always satisfied. This means that some exotic black holes, though having lower entropy than their BTZ counterparts, contain more entropy than their thermodynamic volumes would otherwise naively allow.

Moreover, we study the late time complexity growth for the exotic BTZ black hole using the framework of the conjectured equality Complexity=Action, where the action is calculated in the Wheeler de Witt (WdW) patch. Unlike previous work [31], we take into account the corner terms $[32,33]$. We find that the growth rate for the standard BTZ black hole is as expected, but (somewhat surprisingly) that exotic black holes have no additional contributions to the growth rate. This provides further evidence that the complexity = action conjecture depends on which action one takes into account [34, 35].

\section{Black hole solution from gravitational Chern-Simons}

The Einstein-AdS action in $D=3$ is

$$
I_{\mathrm{EH}}=\frac{1}{8 \pi G_{N}} \int_{M^{3}}\left[e_{a} \wedge R^{a}-\frac{1}{6 \ell^{2}} \epsilon^{a b c} e_{a} \wedge e_{b} \wedge e_{c}\right]
$$

in the well-known Einstein-Cartan formulation, where

$$
T^{a}=d e^{a}+\epsilon^{a b c} \omega_{b} \wedge e_{c}, \quad R^{a}=d \omega^{a}+\frac{1}{2} \epsilon^{a b c} \omega_{b} \wedge \omega_{c}
$$

are the respective torsion and curvature forms, with dreibein 1-forms $e^{a}$ and Lorentz connection 1-forms $\omega^{a}(a=0,1,2)$, and the exterior product of forms is implicit. 
The field equations have the exact solution

$$
d s^{2}=-N^{2} d t^{2}+N^{-2} d r^{2}+r^{2}\left(d \phi+N^{\phi} d t\right)^{2}
$$

with the lapse and the angular shift function equal to

$$
N^{2}=-8 G_{N} m+\frac{r^{2}}{\ell^{2}}+\frac{16 G_{N}^{2} j^{2}}{r^{2}}, N^{\phi}=-\frac{4 G_{N} j}{r^{2}},
$$

where $\ell$ is the $\mathrm{AdS}_{3}$ radius, $G_{N}$ is the $(2+1)$-dimensional Newton constant and $m$ and $j$ are constants of integration, whose interpretation is contingent upon the theory under consideration.

The simplest gravity model for which BTZ black holes are exotic is the parity-odd action for $D=3$ Einstein gravity with $\Lambda<0$ [36]. The action is

$$
I_{\mathrm{GCS}}=\frac{1}{8 \pi G_{N}} \int_{M^{3}}\left[\tilde{\ell} \omega_{a} \wedge\left(d \omega^{a}+\frac{1}{3} \epsilon^{a b c} \omega_{b} \wedge \omega_{c}\right)-\frac{1}{\hat{\ell}} e_{a} \wedge T^{a}\right]
$$

whose parity transform is minus itself (and so its field equations preserve parity). The quantities $\hat{\ell}$ and $\tilde{\ell}$ are coupling constants whose values need not be equal. Varying with respect to $e^{a}$ yields $T^{a}=0$, which in turn allows one to solve for $\omega^{a}$. Inserting the result into the equation obtained by variation with respect to $\omega^{a}$ gives the same field equations as those of the (parity-even) Einstein-AdS action (2.1), with $\ell$ replaced by the geometric mean $\sqrt{\hat{\ell} \tilde{\ell}}$.

An alternative action for obtaining the exotic BTZ black hole is [27]

$$
I_{B C E \omega}=\frac{\ell}{8 \pi G_{N}} \int_{M^{3}}\left(e^{a} \wedge R_{a}[\omega]+B^{a} \wedge D_{\omega} C_{a}\right)
$$

where each of $\left\{e^{a}, \omega^{a}, B^{a}, C^{a}\right\}$ are regarded as independent fields, with $D_{\omega}$ the covariant derivative with respect to the connection $\omega^{a}$. Note that there is no coupling of matter to the metric in (2.6); for this reason the fields $\left\{B^{a}, C^{a}\right\}$ are referred to as topological matter. A variety of solutions exist to the field equations of this theory, one of which is the black hole metric (2.3) [27].

Like a typical rotating black hole solution in $D=4$, the horizon equation for a BTZ black hole $(N=0)$ has two solutions that correspond to two Killing horizons, located respectively at

$$
r_{ \pm}=2 \sqrt{G_{N} \ell\left(\ell m \pm \sqrt{\ell^{2} m^{2}-j^{2}}\right)}
$$

that give the condition $\ell m>|j|$ (necessary to have the two event horizons) and define the extremal case when $\ell m=|j|$. Assuming that $j>0$ then the parameters $m$ and $j$ of the black hole can be expressed as [37]

$$
m=\frac{r_{-}^{2}+r_{+}^{2}}{8 G_{N} \ell^{2}}, j=\frac{r_{-} r_{+}}{4 G_{N} \ell}
$$

However $m$ and $j$ in general are not respectively proportional to the mass and angular momentum of the black hole. This was first pointed out for the action (2.6), where a 
computation of the Noether charges indicated that $M \propto j$ and $J \propto m$, with $M$ and $J$ the respective conserved mass and angular momentum [27]. Setting $\tilde{\ell}=\hat{\ell}=\ell$ for simplicity, more recently it has been noted that $[29,37]$

$$
\begin{gathered}
M=\alpha m+\gamma \frac{j}{\ell}, \\
J=\alpha j+\gamma \ell m,
\end{gathered}
$$

can more generally be considered, with $(\alpha, \gamma)=(1,0)$ corresponding to the normal BTZ black hole, while the case $(\alpha, \gamma)=(0,1)$ corresponds to the exotic BTZ black hole. We shall refer to the class of black holes with general $(\alpha, \gamma)$ as generalized exotic black holes. An analysis of the mass formula for the exotic BTZ black hole in the context of weak cosmic censorship is discussed in [38].

We note here that a possible action that could extrapolate between the standard and the exotic solutions could be defined as the sum of two actions: the standard EinsteinHilbert action (EH) and the Gravitational Chern-Simons action (GCS), as used in [39], where the parameter in front of the latter could play a role analogous to that of an Immirzi parameter [40] in $(2+1)$ dimensions [41, 42]. In other words the following general action $[39,43]$ for the intermediate values of the standard/exotic BTZ black hole would be a linear combination of $I_{\mathrm{EH}}$ and $I_{\mathrm{GCS}}$

$$
\begin{aligned}
I(A)= & \frac{\alpha}{8 \pi G_{N}}\left[\int_{M}\left(e^{a} \wedge R_{a}+\frac{\Lambda}{6} \epsilon_{a b c} e^{a} \wedge e^{b} \wedge e^{c}\right)+\frac{1}{2} \int_{\partial M} K\right] \\
& +\frac{\gamma \ell}{8 \pi G_{N}} \int_{M}\left(\omega^{a} \wedge d \omega_{a}+\frac{1}{3} \epsilon_{a b c} \omega^{a} \wedge \omega^{b} \wedge \omega^{c}+\Lambda e^{a} \wedge T_{a}\right)
\end{aligned}
$$

where $K$ refers to the trace of the second fundamental form at the boundary $\partial M$ and was shown to regularize $(2+1)$ dimensional gravity [44]. The contribution to the WdW patch would therefore be the sum of these contributions (with the proper normalization of the dimensionless $\alpha$ and $\gamma$ coefficients).

The sum can be obtained by recalling that a $(2+1)$ dimensional space of constant negative curvature has symmetry $\mathrm{SO}(2,2)$, for which the generators of the Lie group satisfy [45]

$$
\left[J_{a}, J_{b}\right]=\epsilon_{a b c} J^{c}, \quad\left[J_{a}, P_{b}\right]=\epsilon_{a b c} P^{c}, \quad\left[P_{a}, P_{b}\right]=\frac{1}{\ell^{2}} \epsilon_{a b c} J_{c}
$$

with general invariant quadratic forms

$$
\left\langle J_{a}, J_{b}\right\rangle=\gamma \eta_{a b}, \quad\left\langle J_{a}, P_{b}\right\rangle=\alpha \eta_{a b}, \quad\left\langle P_{a}, P_{b}\right\rangle=\gamma \eta_{a b}
$$

where the constants $\alpha$ and $\gamma$ are introduced in eq. (2.11) and should be fixed to zero respectively if one wants to separately analyze only the Einstein-Hilbert case (EH) or the gravitational Chern Simon (GCS) case.

The shift (2.9), (2.10) was found in $[39,46]$ and is given by the fact that the action (2.11) describes a class of theories with topological mass, where the mass and angular momentum of the BTZ black hole are linear combinations of the mass and angular momentum that 
can be calculated in pure GR. The variation of the total action (2.11) with respect to the metric gives the following equations

$$
R_{\mu \nu}-\frac{1}{2} g_{\mu \nu} R-g_{\mu \nu} \ell+\gamma C_{\mu \nu}=0
$$

where $C_{\mu \nu}$ is the Cotton tensor obtained varying the gravitational Chern-Simons action with respect to the metric.

\subsection{Thermodynamics}

A full treatment of the extended phase space would entail a separate thermodynamic interpretation of $\tilde{\ell}, \hat{\ell}$, and $\ell$ as distinct thermodynamic quantities, each with their own conjugate. The net effect would be to introduce three distinct pressures, each with their own conjugate volume. This will add unnecessary complications to the basic thermodynamics of the generalized exotic BTZ black hole, and so for simplicity we shall consider $\tilde{\ell}=\hat{\ell}=\ell$ henceforth.

The Hawking temperature and the angular velocity associated with the event horizon read

$$
T=\frac{r_{+}}{2 \pi \ell^{2}}-\frac{8 G_{N}^{2} j^{2}}{\pi r_{+}^{3}}, \quad \Omega=4 \frac{G_{N} j}{r_{+}^{2}},
$$

and are obtained from (2.3) using the horizon equation $N=0$. These expressions for these intensive thermodynamic variables are geometric and model independent [29], i.e. independent of the particular field equations that are solved by the BTZ metric. They only depend on the position of the Killing horizon.

From (2.9) and (2.10) we see that the extensive thermodynamic variables are modeldependent. Furthermore, the system of equations (2.9), (2.10) is singular when $\alpha=\gamma$. However, in the particular case $\ell m=j$ (that is the extremal solution) the condition $\ell M=J$ also holds for every value of $\alpha$ and $\gamma$ (including $\alpha=\gamma$ ). Likewise if $\alpha=\gamma$ then $\ell M=J$, regardless of the distinct values of $m$ and $j$. Rewriting the condition $\ell m \geq j$ in terms of the new variables $(M, J)$ we obtain two inequalities

$$
\left\{\begin{array}{l}
\ell M \geq J, \quad \alpha \geq \gamma \\
\ell M \leq J, \quad \alpha \leq \gamma
\end{array}\right.
$$

and the condition needed to find the horizon is different for each. This general analysis is valid for the redefinition of the conserved charges in eqs. (2.9), (2.10) and it is not necessary to have an extended phase space. In figure 1 we show the behaviour of the temperature for the two different situations.

\subsection{Extended phase space}

Based on the hypothesis (1.3), we now add to the intensive variables (2.15) another intensive quantity

$$
P=\frac{1}{8 \pi G_{N} \ell^{2}}
$$



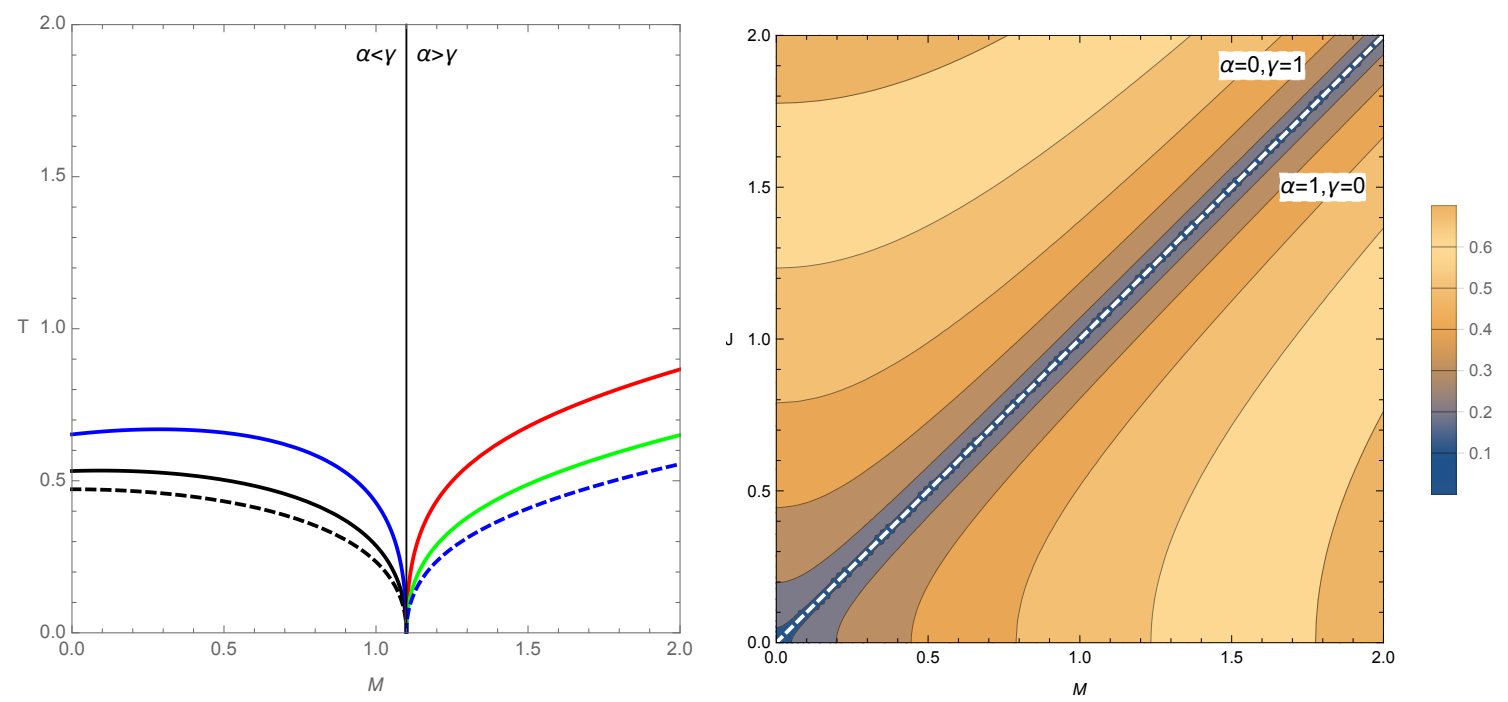

Figure 1. Left: the two different sectors of the $(T, M)$ parameter space are divided by the vertical black line, which corresponds to the extremal case $\ell M=J$. The area on the left $(\alpha<\gamma)$ admits the exotic solution $\gamma=1, \alpha=0$ (black dashed line) for $J=1.1$ whereas the area on the right $(\alpha>\gamma)$ admits the standard BTZ solution $\gamma=0, \alpha=1$ (blue dashed line) for $J=1.1$. The black/blue solid lines at the left are the respective cases $(\alpha=0.2, \gamma=0.6)$ and $(\alpha=0.4, \gamma=0.6)$, whereas the red and green solid lines respectively correspond to $(\alpha=0.6, \gamma=0.4)$ and $(\alpha=0.8, \gamma=0.2)$. Right: contour plot of the temperature as function of $J$ and $M$ for the two sectors: the exotic and the standard case.

that is only geometric and model independent. The first law of black hole thermodynamics (1.1) is

$$
d M=T d S+V d P+\Omega d J
$$

and we wish to check this law for general values of $(\alpha, \gamma)$.

Inserting (2.8) in the previous general definitions $(2.9),(2.10)$ of $(M, J)$ that interpolate between the classical and the exotic BTZ black hole we obtain

$$
\begin{gathered}
M=\frac{\alpha\left(r_{-}^{2}+r_{+}^{2}\right)}{8 G_{N} \ell^{2}}+\frac{\gamma r_{-} r_{+}}{4 G_{N} \ell^{2}}, \\
J=\frac{\alpha r_{-} r_{+}}{4 G_{N} \ell}+\frac{\gamma\left(r_{-}^{2}+r_{+}^{2}\right)}{8 G_{N} \ell} \\
T=\frac{r_{+}^{2}-r_{-}^{2}}{2 \pi \ell^{2} r_{+}} \quad \Omega=\frac{r_{-}}{r_{+} \ell}
\end{gathered}
$$

Since $M$ is a function of $\left(r_{+}, r_{-}, \ell\right)$ then

$$
\begin{aligned}
d M\left(r_{+}, r_{-}, \ell\right) & =\left(\frac{\partial M}{\partial r_{-}}\right) d r_{-}+\left(\frac{\partial M}{\partial r_{+}}\right) d r_{+}+\left(\frac{\partial M}{\partial \ell}\right) d \ell \\
& =\left(\frac{\alpha r_{-}}{4 G_{N} \ell^{2}}+\frac{\gamma r_{+}}{4 G_{N} \ell^{2}}\right) d r_{-}+\left(\frac{\alpha r_{+}}{4 G_{N} \ell^{2}}+\frac{\gamma r_{-}}{4 G_{N} \ell^{2}}\right) d r_{+}+\left(-\frac{\alpha\left(r_{-}^{2}+r_{+}^{2}\right)}{4 G_{N} \ell^{3}}-\frac{\gamma r_{-} r_{+}}{2 G_{N} \ell^{3}}\right) d \ell \\
& =\frac{1}{4 G_{N} \ell^{2}}\left[\left(\alpha r_{-}+\gamma r_{+}\right) d r_{-}+\left(\alpha r_{+}+\gamma r_{-}\right) d r_{+}\right]+\left(-\frac{\alpha\left(r_{-}^{2}+r_{+}^{2}\right)}{4 G_{N} \ell^{3}}-\frac{\gamma r_{-} r_{+}}{2 G_{N} \ell^{3}}\right) d \ell
\end{aligned}
$$


Expanding the right-hand-side of (2.18), we have

$$
\begin{aligned}
T d S & =\frac{r_{+}^{2}-r_{-}^{2}}{2 \pi \ell^{2} r_{+}}\left[\left(\frac{\partial S}{\partial r_{-}}\right) d r_{-}+\left(\frac{\partial S}{\partial r_{+}}\right) d r_{+}\right] \\
\Omega d J & =\frac{\Omega}{4 G_{N} \ell}\left[\left(\gamma r_{-}+\alpha r_{+}\right) d r_{-}+\left(\alpha r_{-}+\gamma r_{+}\right) d r_{+}-\frac{\gamma r_{-}^{2}+2 \alpha r_{-} r_{+}+\gamma r_{+}^{2}}{2 \ell} d \ell\right] \\
V d P & =V\left(-\frac{1}{4 \pi G_{N} \ell^{3}}\right) d \ell
\end{aligned}
$$

where $\Omega=r_{-} /\left(\ell r_{+}\right)$and we suppose that the entropy has no dependence from the cosmological constant $S\left(r_{+}, r_{-}\right)$.

Equating the terms for $d r_{+}, d r_{-}$and $d \ell$ in (2.18), yields

$$
\frac{\partial S}{\partial r_{+}}=\frac{\pi \alpha}{2 G_{N}} \quad \frac{\partial S}{\partial r_{-}}=\frac{\pi \gamma}{2 G_{N}}
$$

and so

$$
\begin{aligned}
& S=\frac{1}{2 G_{N}}\left(\pi \alpha r_{+}+\pi \gamma r_{-}\right) \\
& V=\alpha \pi r_{+}^{2}+\gamma \pi r_{-}^{2}\left(\frac{3 r_{+}}{2 r_{-}}-\frac{r_{-}}{2 r_{+}}\right)
\end{aligned}
$$

for the entropy and volume respectively. For the standard BTZ black hole $(\gamma=0, \alpha=1)$ we obtain $V=\pi r_{+}^{2}$ (which happens to be the geometric volume of the black hole), while in the exotic case $(\gamma=1, \alpha=0)$ we get a mixed term that depends on both the inner and outer radii. The larger class of generalized exotic BTZ black holes therefore furnish another example of thermodynamic volume that is different from the standard geometric volume. Note that in the extremal case $\left(r_{+}=r_{-}=r_{e x t}\right)$ the volume is $V=\pi r_{e x t}^{2}(\alpha+\gamma)$. It coincides with the geometric volume for $(\alpha+\gamma)=1$, which includes both the standard $(\alpha=1, \gamma=0)$ and exotic $(\alpha=0, \gamma=1)$ cases.

Using the above relations it is straightforward to verify that the Smarr relation in $D=3$ dimensions

$$
0=T S-2 P V+\Omega J
$$

also holds. We pause to comment that it is possible to obtain a general relationship between these quantities that

$$
x M=T S+y \Omega J+z P V
$$

where $\{x, y, z\}$ are constants that obey

$$
x-z=2 \quad 2 y-z=4
$$

for all $(\alpha, \gamma)$. More general relations of the type in equation (2.30) are sometimes referred to as Smarr relations [47, 48], but they do not respect the correct Euler scaling relations unless $x=0$, forcing $y=1$ and $z=-2$, yielding (2.29). 
At this point, we can make two interesting considerations:

- We required the validity of the laws (2.18) and (2.29) of standard black hole thermodynamics to also hold in the case of the exotic black hole. This requirement gave us the correct entropy for the exotic BTZ black hole [27, 29, 39]:

$$
I_{E}=\frac{\pi r_{-}}{2 G_{N}}
$$

- The thermodynamic volume depends on both variables $(\alpha, \gamma)$; in the exotic case it becomes

$$
V=\pi r_{-}^{2}\left(\frac{3 r_{+}}{2 r_{-}}-\frac{r_{-}}{2 r_{+}}\right)
$$

The volume is positive when the following condition is satisfied

$$
r_{+}^{2}\left(2 \alpha r_{+}+3 \gamma r_{-}\right)>\gamma r_{-}^{3}
$$

that in the case $\alpha=0$ becomes $r_{+}>r_{-} / \sqrt{3}$. It is always satisfied for the standard BTZ black hole, for which $\gamma=0$.

So far our analysis holds for general values of $(\alpha, \gamma)$. For the remainder of this paper we shall assume normalization of the parameters such that $\alpha+\gamma=1$.

\section{Pressure-volume behaviour}

It is possible to solve (2.20) and (2.28) for both $r_{+}$and $r_{-}$in terms of $(P, V, J)$. Inserting the results into $(2.21)$ we can solve for $P$ in terms of $(T, V, J)$ and obtain an equation of state for the generalized exotic black hole.

We can preliminarily explore the expected $P V$ diagram characteristics of these systems by considering the small and large $J$ limits of the pure exotic and normal black hole equations of state. Ignoring constants for the time being, setting $(\alpha=1, \gamma=0)$ the equation of state takes the simple dependence

$$
P \sim \frac{J^{2}}{V^{2}}+\frac{T}{\sqrt{V}}
$$

which strictly decreases for increasing $V$. Particular values of $J$ and $T$ have no effect on the general qualitative shape of the corresponding $P V$ diagram.

In the pure exotic limit $(\alpha=0, \gamma=1)$, we find a much more complicated expression that exhibits a maximum volume when $J \gg T$. Although the full expression is not trivial to solve, we can expand $V$ as a series in $T$ to third order, which gives

$$
V \sim \frac{\sqrt{2.51 G_{N}} J}{\sqrt{P}}+\frac{0.351 T \sqrt{J}}{\left(G_{N} P\right)^{3 / 4}}-\frac{0.0491 T^{2}}{G_{N}^{2} P^{2}}
$$

which reaches a maximum when

$$
P \sim \frac{0.138 T^{4 / 3}}{G_{N}^{5 / 3} J^{2 / 3}}
$$



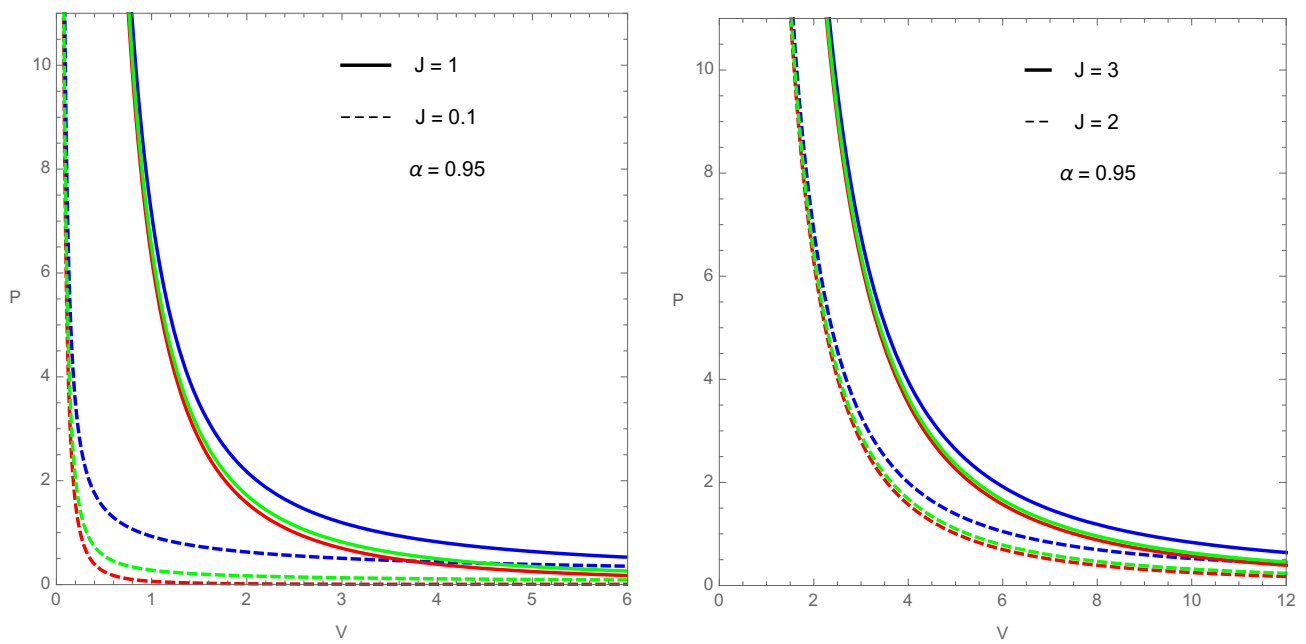

Figure 2. PV diagram for $\alpha=0.95, \gamma=0.05, J=0.1,1$ (left) and $J=2,3$ (right), and $T=0.001$ (red), $T=0.5$ (green), $T=2$ (blue).

In this case, it becomes possible for the system to admit two pressures for the same thermodynamic volume. For small $J$, one can verify the volume behaves as $V \sim P^{-2}$. Also, it can be shown that the angular momentum $J$ has a lower bound, which generalizes to arbitrary $\gamma$ as

$$
J \geq \frac{1}{32} \sqrt{\frac{\pi}{2}} \sqrt{\frac{T^{4} \gamma^{2}}{G_{N}^{5} P^{3}}} .
$$

The full equation of state for general $\alpha$ and $\gamma$ is analytically cumbersome. Rather than give the explicit expressions, we shall compute the $P V$ diagrams parametrically. Specifically, we focus on the trends in the implicit plots as the black holes go from being "normal" $(\alpha=1)$ to exotic $(\alpha=0)$, as well as for increasing angular momentum $J$ and temperature $T$.

To construct the plots we solve the system of equations (2.20) and (2.21) for both $r_{+}$ and $r_{-}$, inserting the results in the definition of the thermodynamic volume (2.33) in order to obtain the $P V$ plot. There are two different solutions depending on whether $\alpha>\gamma$ or $\alpha<\gamma$. The cuts in the following plots are given by the above lower bound on $J$.

Figure 2 illustrates the characteristics of a $95 \%$ regular black hole $(\alpha=0.95, \gamma=0.05)$ for increasing $J$. Each curve is isothermal, with the temperature increasing from left to right. There is no apparent critical behaviour, nor any change in the general shape of the curves, with only their concavity decreasing as $J$ grows. Figure 3 depicts this relation for the same parameters for a $75 \%$ regular $(\alpha=0.75)$ black hole. There is no significant deviation between the three cases.

Interesting behaviour begins to emerge for smaller $\alpha$. In figure 4 , for which $\alpha=0.30$, we see that in the limit of small $V$, the isothermal curves become double-valued. This actually happens for the higher- $\alpha$ black holes as well, though it is at a much smaller volume scale as compared to the more exotic black holes. In fact, as $J$ increases the overlap region of the isothermal curves becomes increasingly large (see figure 5 ). This suggests that the angular 

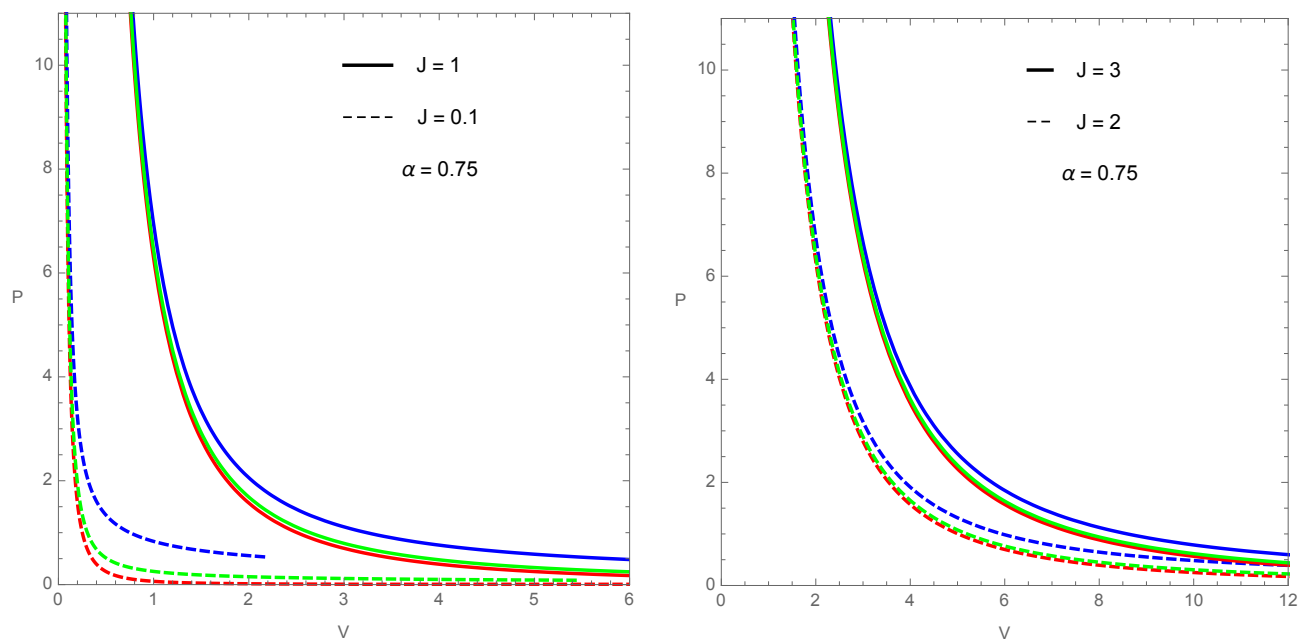

Figure 3. PV diagram for $\alpha=0.75, \gamma=0.25, J=0.1,1,2,3$, and $T=0.001$ (red), $T=0.5$ (green), $T=2$ (blue). In the first panel it is possible to see where the solution that satisfies eq. (2.16) ceases to exist.
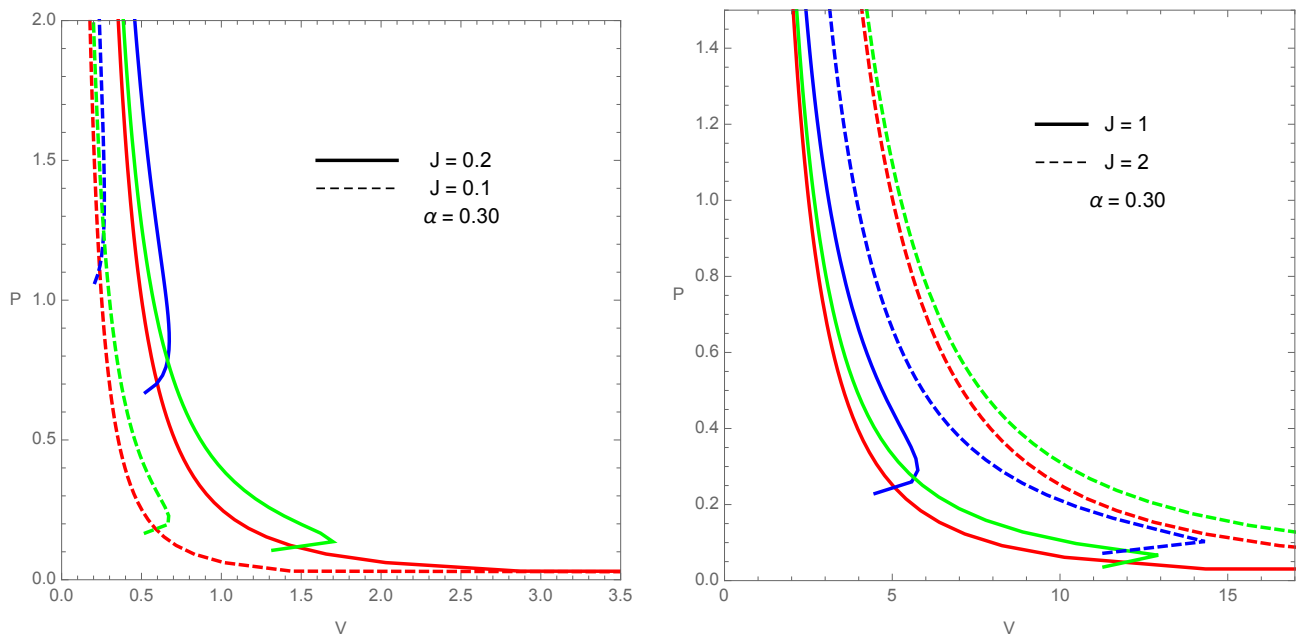

Figure 4. PV diagram for $\alpha=0.30, \gamma=0.70, J=0.1,0.2,1,2$, and $T=0.001$ (red), $T=0.5$ (green), $T=2$ (blue).

momentum of the black holes plays a much more significant role in the chemistry of exotic black holes, as compared to the normal ones.

In the case where $\alpha=0$, i.e. a purely exotic BTZ black hole, the phase plots show similar behaviour as above, only more pronounced (see figure 5). For sufficiently large $T$ and small $J$, the associated pressure is a smoothly increasing function of $V$. As temperature decreases, a critical value is reached at which the curve becomes double-valued - for small enough $V$ two states can exist with different pressures. For large enough $J$ and small enough $T$ this double-valuedness is persistent. As $V \rightarrow 0$ the pressure remains finite. 

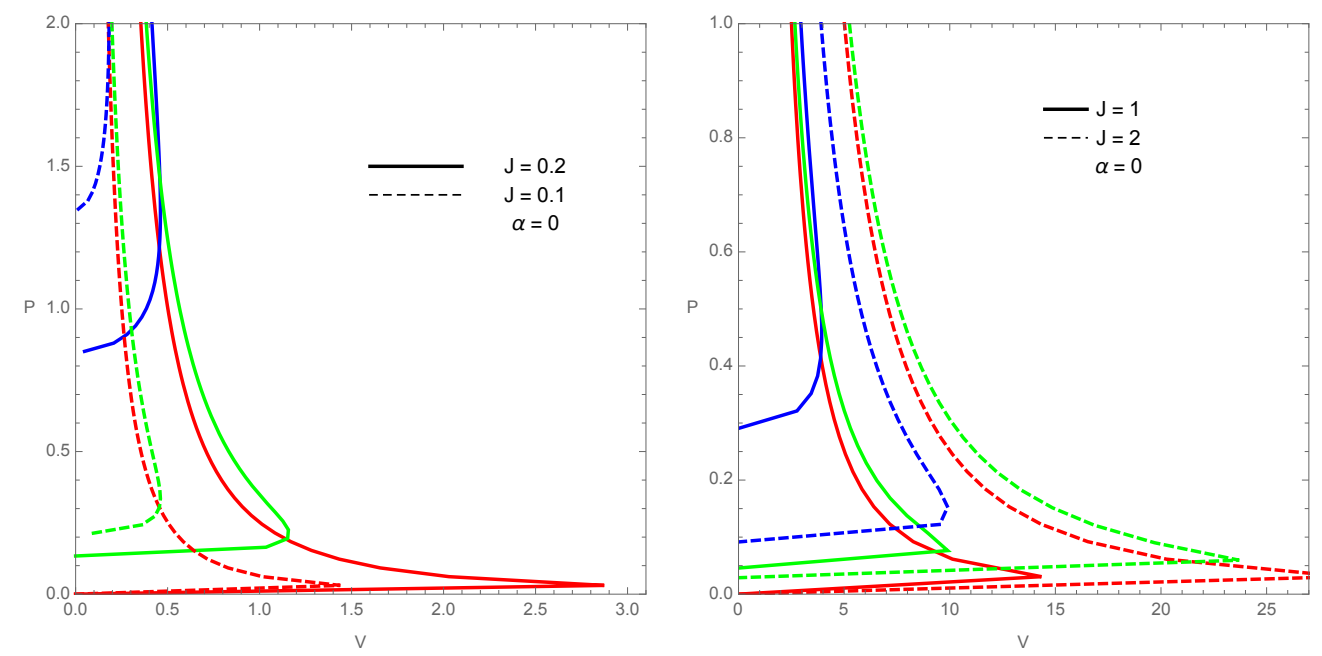

Figure 5. PV diagram for $\alpha=0, \gamma=1$ (pure exotic BTZ black hole). The other parameters are $J=0.1,1,2,3$, and $T=0.001$ (red), $T=0.5$ (green), $T=2$ (blue). In the case of exotic BTZ there is a value of the pressure for which the volume is equal to zero.

\section{Reverse isoperimetric ratio}

One quantity of interest for black holes is the reverse isoperimetric inequality [30], which is the conjecture that

$$
\mathcal{R}=\left(\frac{(D-1) V}{\omega_{D-2}}\right)^{\frac{1}{D-1}}\left(\frac{\omega_{D-2}}{A}\right)^{\frac{1}{D-2}} \geq 1
$$

for any asymptotically AdS black hole, with $A$ identified with the horizon area, $V$ with the associated thermodynamic volume, and $\omega_{D-2}=2 \pi^{\frac{D-1}{2}} / \Gamma\left(\frac{D-1}{2}\right)$. For Schwarzschild-AdS black holes the bound is saturated, which means that for a fixed thermodynamic volume the entropy of the black hole is maximized for Schwarzschild-AdS spacetime.

For generalized exotic BTZ black holes we obtain

$$
\mathcal{R}=\frac{1}{2} \sqrt{\frac{4 \alpha r_{+}^{3}-2 \gamma r_{-}^{3}+6 \gamma r_{-} r_{+}^{2}}{r_{+}^{3}}}
$$

which is saturated for the BTZ case $(\alpha=1, \gamma=0)$ but is otherwise violated for all other values of $(\alpha, \gamma)$. We illustrate this in figure 6 .

\section{Gibbs free energy and critical behaviour}

The Gibbs free energy of the black holes can be readily calculated, and the corresponding plots for different degrees of exoticness are displayed in figures 7 and 8 . The plots are obtained solving (2.20) with respect to $r_{-}$and substituting it in the temperature (2.21) and in the Gibbs free energy

$$
G=M-T S=\frac{3 \alpha r_{+} r_{-}^{2}-\alpha r_{+}^{3}+2 \gamma r_{-}^{3}}{8 G_{N} \ell^{2} r_{+}}
$$




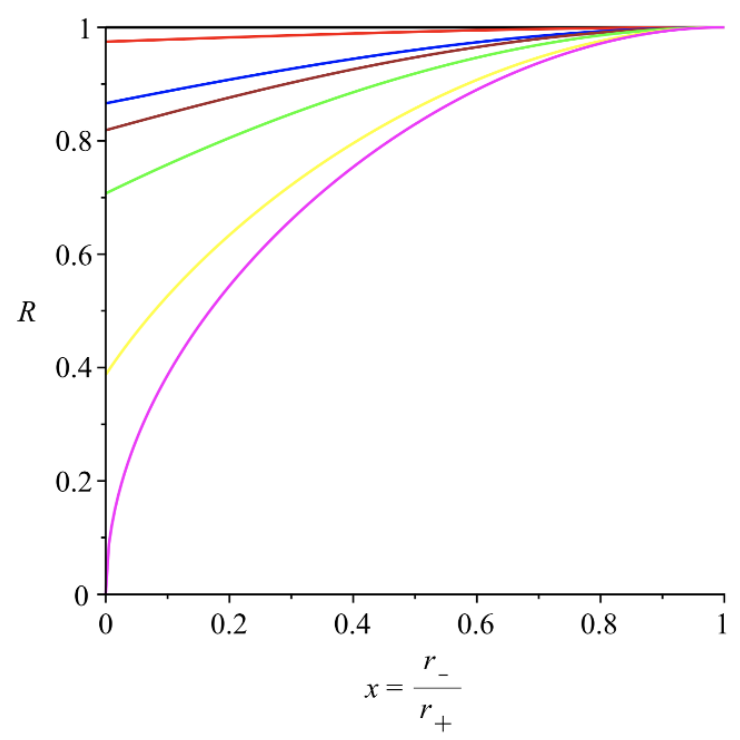

Figure 6. Reverse isoperimetric ratio plotted as a function of $x=r_{-} / r_{+}$for various values of $(\alpha, \gamma):(1,0)$ (black), $(.95, .05)$ (red), $(.75, .25)$ (blue), $(.67, .33)$ (brown), $(0.5,0.5)$ (green) $(.15, .85)$ (yellow), $(0,1)$ (magenta).

There is a stark distinction between the cases where $\alpha>0.5$ (majority standard) and $\alpha<0.5$ (majority exotic). For $\alpha<\gamma$, there is an upper bound for $r_{+}$such that $r_{E} \leq r_{+} \leq r_{+_{M}}$ where

$$
r_{E}=2 \sqrt{G_{N} J \ell} \quad r_{+_{M}}=2 \sqrt{2} \sqrt{\frac{\gamma G_{N} J \ell}{\gamma-\alpha}}
$$

are respectively obtained by solving (2.20) with respect to $r_{-}$and $T=0$ with respect to $r_{+}$(so that $r_{E}$ is the same both in the standard and exotic cases) and using the relation $(\alpha+\gamma)=1$.

As shown in figure 7, the concavity of the two cases is opposite. Moreover, the free energy of the purely exotic black hole terminates in $r_{+_{M}}$ to zero for increasing temperature, while that of the standard black hole can be negative and is unbounded from below.

The intermediate cases where $0<\alpha, \gamma<1$ reveal even more interesting features for the majority exotic case. As figure 8 indicates, the energy curve reaches a minimum, but can assume two different values for the same temperature. This behaviour is most pronounced for the transitional case at $\alpha \rightarrow 0.5$ with $\gamma=1-\alpha$, and abates as $\alpha \rightarrow 0$. For $\alpha<\gamma$, the final points in figures 7 and 8 are

$$
\begin{aligned}
T\left(r_{+_{M}}\right) & =\frac{\sqrt{2} \sqrt{\gamma G_{N} J \ell(\gamma-\alpha)}}{\pi \gamma^{2} \ell^{2}} \\
G\left(r_{+_{M}}\right) & =-\frac{\alpha J}{\gamma \ell}
\end{aligned}
$$

Local thermodynamic stability is given by positivity of the specific heat, defined as

$$
C_{P}=T\left(\frac{\partial S}{\partial T}\right)_{P, J}
$$




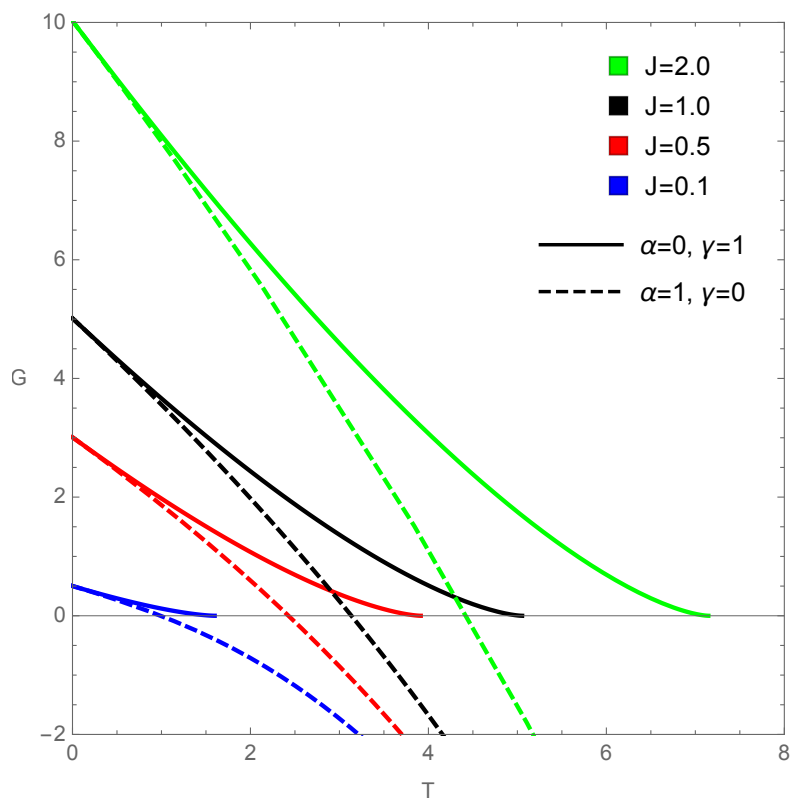

Figure 7. Gibbs free energy for a standard ( $\alpha=1 ; \gamma=0$, dashed curve) and a purely exotic black hole $(\alpha=0 ; \gamma=1$, solid curve), for angular momentum values $J=2.0$ (green), 1.0 (black), 0.5 (red), and $J=0.1$ (blue) and $P=1$.

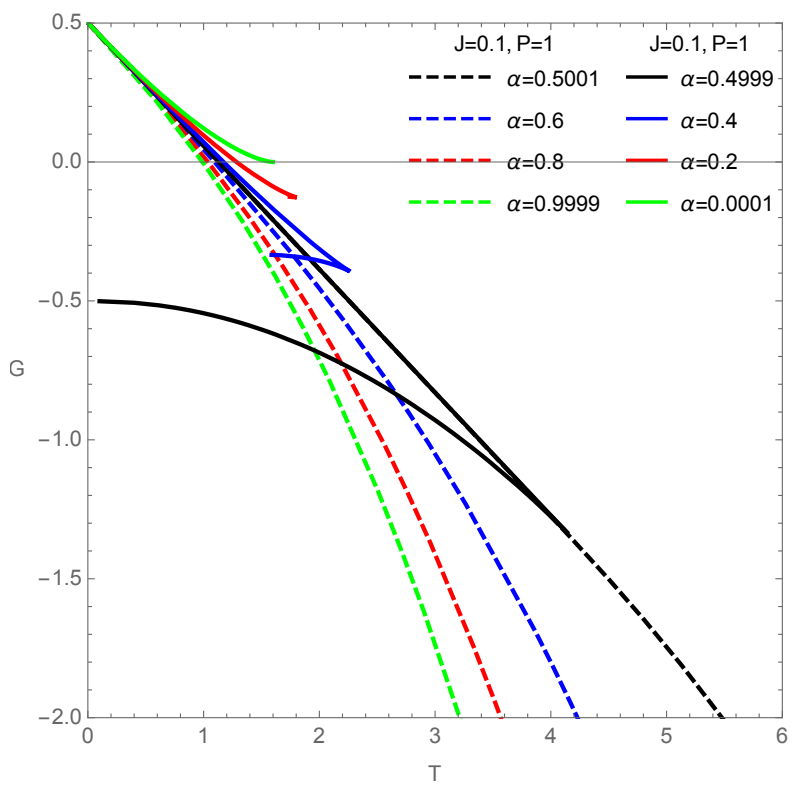

Figure 8. Gibbs free energy at the transition between majority standard $(\alpha>0.5 ; \gamma<0.5)$ and majority exotic $(\alpha<0.5 ; \gamma>0.5)$ black holes.

Since the temperature has two different behaviours in the two different situations $\alpha>\gamma$ or $\gamma>\alpha$, we analyze the two cases separately.

For $\alpha>\gamma$ and $(\alpha=1, \gamma=0)$ the specific heat is always positive (dashed lines in figure 9$)$. The pure exotic case $(\alpha=0, \gamma=1)$ always has negative specific heat therefore is locally unstable (see right panel in figure 9). However, for intermediate values of $\alpha$ and $\gamma$ 

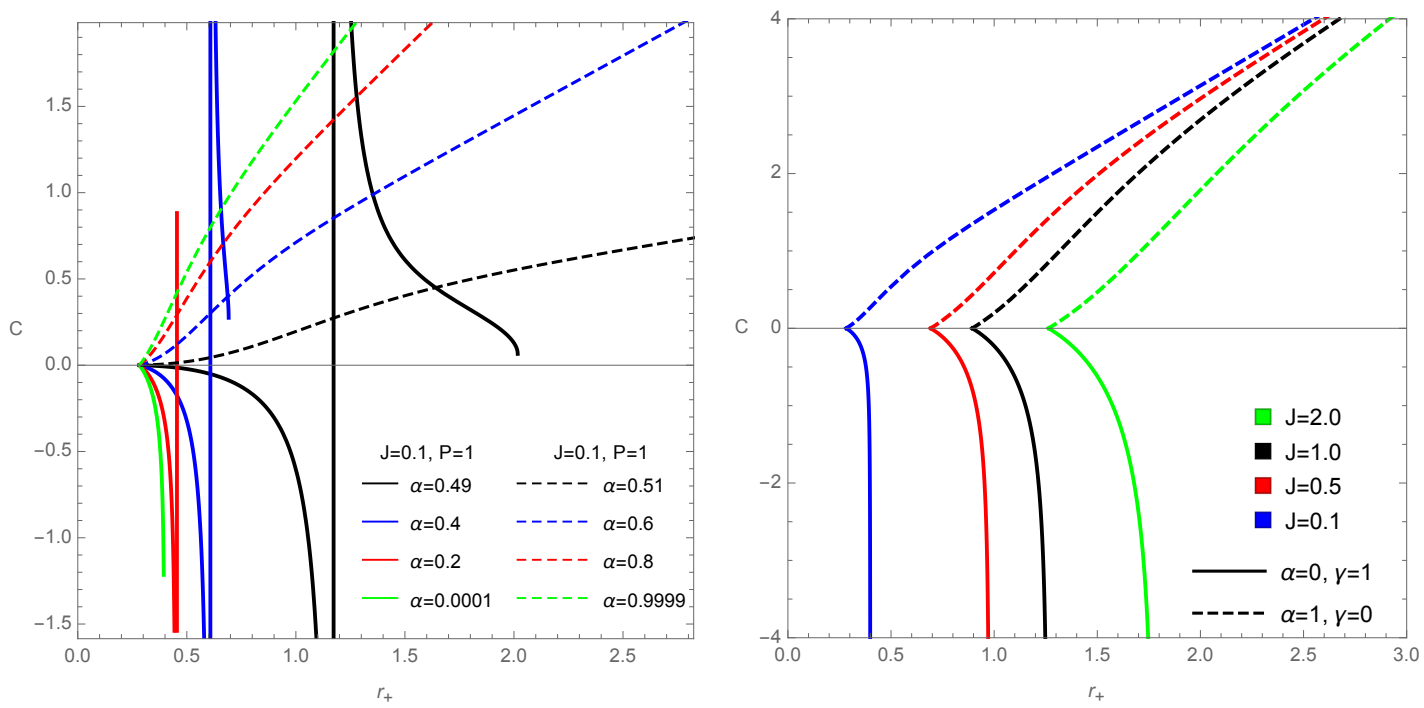

Figure 9. Specific heat: Left panel: different $\alpha$ 's and respective $\gamma=1-\alpha$. Right panel: pure exotic and standard case for several values of $\mathrm{J}$.

(with $\alpha<\gamma$ ) there are two branches (left panel in figure 9), and the one with lower Gibbs free energy has positive specific heat while the other one has negative specific heat. The black holes with positive specific heat can be in stable equilibrium at a fixed temperature.

\subsection{Free energy with fixed- $\Omega$}

In the previous sections, we studied the canonical ensemble with fixed angular momentum but free horizon angular velocity. Now, we consider the grand-canonical ensemble with the fixed angular velocity of the horizon and free angular momentum.

$$
G_{\Omega}=M-T S-\Omega J=G_{\Omega}(T, P, \Omega)
$$

Using eqs. (2.21), we can write $r_{-}=\ell r_{+} \Omega$ and substitute it in the temperature that reads

$$
T=\frac{r_{+}\left(1-\ell^{2} \Omega^{2}\right)}{8 \ell^{2} G_{N}}
$$

and therefore, the temperature is positive if $0<\Omega<1 / \ell$ while $\Omega=1 / \ell$ gives the extremal case $T=0$ (the same is true if we express the temperature as function of $r_{-}$). The Gibbs free energy (5.6) as function of $T$ and $P$ is

$$
G_{\Omega}=-\frac{\pi T^{2}\left(\sqrt{2 \pi} \gamma \Omega+4 \pi \alpha \sqrt{G_{N} P}\right)}{8 G_{N}^{3 / 2} \sqrt{P}\left(8 \pi G_{N} P-\Omega^{2}\right)}
$$

and it is positive iff $\Omega>1 / \ell$, that, as we said before, it is not allowed. Consequently, $G_{\Omega}$ will always be negative for $r_{+}>0$. See the right panel in figure 10 for different values of $\alpha$ and $\Omega$.

In particular, in the exotic limit, the free energy reads

$$
G_{\Omega}(\alpha=0, \gamma=1)=-\frac{\pi^{3 / 2} T^{2} \Omega}{4 \sqrt{2} G_{N}^{3 / 2} \sqrt{P}\left(8 \pi G_{N} P-\Omega^{2}\right)}
$$



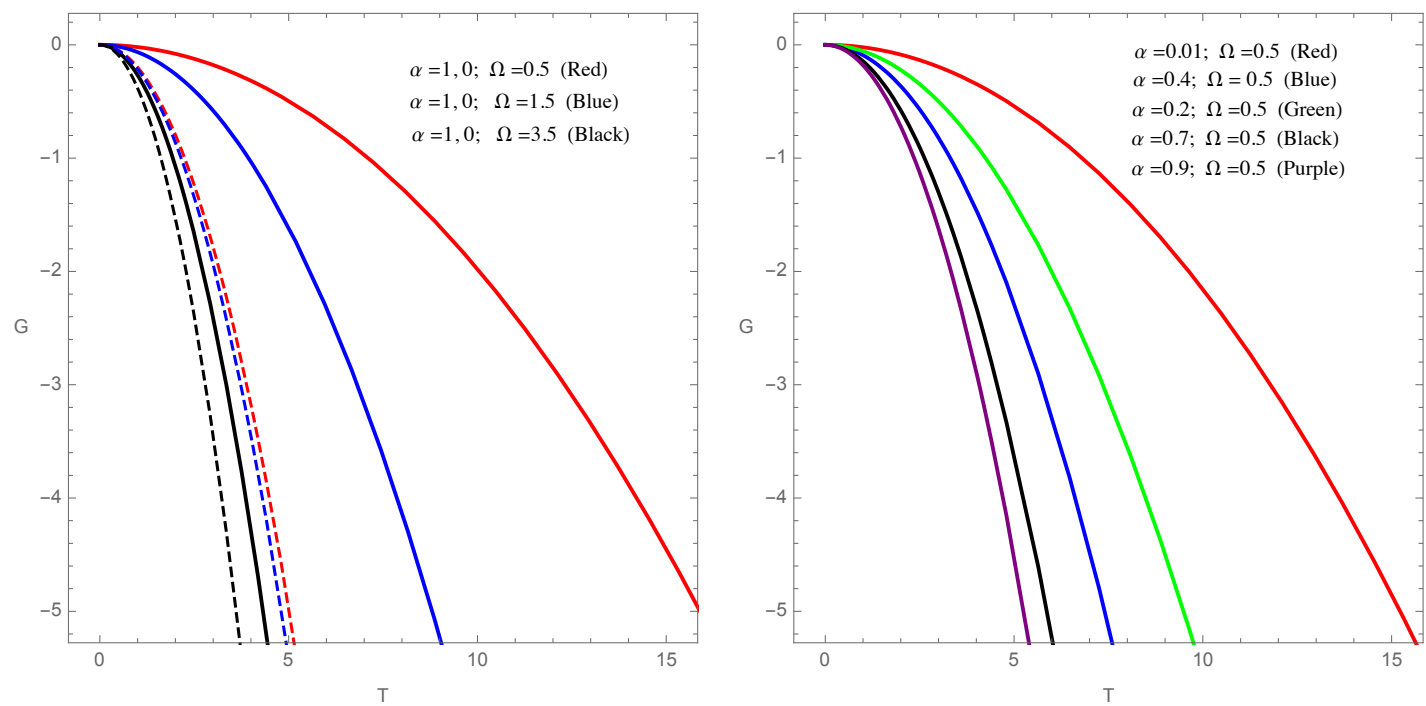

Figure 10. Gibbs free energy for the grand-canonical ensemble: Left panel: Pure exotic (continuous lines) and standard case (dashed lines) for several values of $\Omega$. Right panel: different $\alpha$ 's and respective $\gamma=1-\alpha$.

and in the standard case

$$
G_{\Omega}(\alpha=1, \gamma=0)=-\frac{\pi^{2} T^{2} \sqrt{G_{N} P}}{2 G_{N}^{3 / 2} \sqrt{P}\left(8 \pi G_{N} P-\Omega^{2}\right)} .
$$

The left panel in figure 10 shows the function $G_{\Omega}$ in the exotic and the standard case for different values of $\Omega$.

\section{Complexity growth rate}

Given the interplay between mass and angular momentum that the exotic BTZ black holes show, we wish to check if the bound on complexity growth introduced in [49] is valid or violated. In the $\mathrm{AdS} / \mathrm{CFT}$ correspondence, the complexity $\mathcal{C}$ of a particular state $|\psi\rangle$ can be related to a spacetime region in the bulk. In particular, the complexity=action proposal or the $\mathcal{C} \mathcal{A}$-duality states that

$$
\mathcal{C}\left(\left|\psi\left(t_{L}, t_{R}\right)\right\rangle\right)=\frac{\mathcal{A}}{\pi \hbar}
$$

where $t_{L}$ and $t_{R}$ are, respectively, the time on the left and the right boundary and $\mathcal{A}$ is the bulk action evaluated on the Wheeler-DeWitt (WdW) patch (see figure 11). From this duality, it follows that at late-time the complexity approaches the black hole mass in the following way

$$
\lim _{t_{L} \rightarrow \infty} \frac{d \mathcal{C}}{d t_{L}}=\frac{2 M}{\pi \hbar}
$$

This seems to agree with the Lloyd bound, which limits from above the complexity by the energy

$$
\dot{\mathcal{C}} \leq \frac{2 E}{\pi \hbar} .
$$


The connection between the Schwarzschild-AdS WdW patch with the space-time volume and complexity has been pointed out in [50]. It has notably been showed that the thermodynamic volume and the pressure seems to emerge naturally from the late-time rate of growth (see appendix B for more discussions about the connection between thermodynamic volume and complexity).

In the standard thermodynamic phase-space, it has been shown [49] that in the case of conserved angular momentum for a BTZ black hole, the complexification bound is

$$
\frac{d \mathcal{C}}{d t} \leq \frac{2}{\pi \hbar}\left[(M-\Omega J)-(M-\Omega J)_{g s}\right]
$$

as computed from the Einstein-Hilbert action with Gibbons-Hawking term and corner terms (see also appendix A for more details). The subscript " $g s "$ refers to the "ground state", i.e. the state of lowest $(M-\Omega J)$ for a given angular momenta. The ground state is there in order to obtain a zero complexification rate for the extremal black hole (that has zero temperature). Therefore the ground state could either be an extremal black hole or empty AdS.

For the BTZ black hole

$$
M-\Omega J=\frac{r_{+}^{2}-r_{-}^{2}}{8 G \ell^{2}}=\sqrt{M^{2}-\frac{J^{2}}{\ell^{2}}}
$$

which saturates the complexification bound (6.4) [49]. The rate of growth of the action for the rotating BTZ black hole is

$$
\frac{d \mathcal{A}}{d t}=\frac{r_{+}^{2}-r_{-}^{2}}{4 G_{N} \ell^{2}}=2 \sqrt{M^{2}-\frac{J^{2}}{\ell^{2}}}
$$

that shows that the rotating BTZ black hole saturates the bound (6.4). The ground state for a standard rotating BTZ does not contribute because has $M=0=J$ at fixed $\Omega$.

In the exotic case the previous bound cannot be automatically valid since for $\alpha<\gamma$ follows $\ell M<J$. We shall compute it from the action (2.5) and compare it to the corresponding result for the BTZ case, computed using (2.1). Some of the useful calculations needed to calculate complexity growth for rotating BTZ black holes can be found in appendix A. In particular, using eq. (A.22) in eq. (6.4) it is possible to see that, in the standard BTZ case, $d \mathcal{C} / d t \propto T S[51]$. In the BTZ case one also has $d \mathcal{C} / d t \propto P\left(V_{+}-V_{-}\right)$where $V_{-}=\pi r_{-}^{2}$ and $V_{+}=\pi r_{+}^{2}$ are the BTZ thermodynamic volumes of the inner horizon and the outer horizon respectively [50].

\subsection{Complexity of the exotic BTZ black hole}

The parity-odd action for $D=3$ Einstein gravity with $\Lambda<0$ is given by (2.5) [36]

$$
I_{\mathrm{GCS}}=\frac{\ell}{8 \pi G} \int_{M^{3}}\left[\omega_{a} \wedge\left(d \omega^{a}+\frac{1}{3} \epsilon^{a b c} \omega_{b} \wedge \omega_{c}\right)-\frac{1}{\ell^{2}} e_{a} \wedge T^{a}\right]
$$

Note that contrary to what happens in the case of the EH action, we don't need a boundary term to add to this action in order to have a well defined entropy and variational principle (see, e.g., [39]); we discuss other possible boundary terms in appendix B. 
The solution to the equations of motion from the action (6.7) is given by the space-time triad $[27]$

$$
\begin{aligned}
e^{0} & =\sqrt{\nu^{2}(r)-1}\left(\frac{r_{+}}{\ell} d t-r_{-} d \phi\right) \\
e^{1} & =\frac{\ell}{\nu(r)} d\left[\sqrt{\nu^{2}(r)-1}\right]=\frac{\ell}{\nu(r)}\left[\frac{\nu(r) \nu^{\prime}(r)}{\sqrt{\nu^{2}(r)-1}}\right] d r \\
e^{2} & =-2 \nu(r)\left(\frac{r_{+}}{\ell^{2}} d t-\frac{r_{-}}{\ell} d \phi\right)
\end{aligned}
$$

with $\nu^{2}(r):=r^{2}-r_{-}^{2} / r_{+}^{2}-r_{-}^{2}$ and the prime is the derivative with respect to $r$. The compatible spin connection has the following components:

$$
\begin{aligned}
\omega^{0} & =-2 \sqrt{\nu^{2}(r)-1}\left(\frac{r_{+}}{\ell} d \phi-\frac{r_{-}}{\ell^{2}} d t\right) \\
\omega^{1} & =0 \\
\omega^{2} & =-2 \nu(r)\left(\frac{r_{+}}{\ell^{2}} d t-\frac{r_{-}}{\ell} d \phi\right)
\end{aligned}
$$

and the torsion $T^{a}=0$.

It is straightforward to see that the action (6.7) vanishes for the solution (6.8) (with (6.9)) since $T^{a}=0$ and $\omega^{1}=0$. The latter implies that $\epsilon^{a b c} \omega_{a} \wedge \omega_{b} \wedge \omega_{c}=0$. Furthermore, $d \omega^{0} \propto d r \wedge \omega^{0}$ and $d \omega^{2} \propto d r \wedge \omega^{2}$, so the first term in (6.7) also vanishes. Therefore there is no contribution from the action (6.7) evaluated in the WdW patch $\mathcal{V}$ (see left panel in figure 11 and eq. (A.3)) and we are left only with the boundary and the joint contributions from (2.11); we evaluate these in appendix A.

Now we consider possible boundary terms for the exotic case. Boundary terms for Chern-Simons theory have been previously discussed in literature (see for example [52-55]. In this particular case of a rotating exotic BTZ black hole, as in the non-exotic case, there is no boundary term in the WDW patch that needs to be evaluated on a space-like surface (see the left panel in figure 11). Therefore we will be interested here only about the boundary terms that should be evaluated on the null-like surfaces.

We know that the variation of the total action $\left(I_{\mathrm{EH}}+I_{\mathrm{GCS}}\right)$ with respect to the metric is well-defined if only the GHY boundary term is included [39], up to a counterterm ambiguity $[37,43,56]$ that we discuss in appendix B. Using this formulation then one can say that there are no other corner terms to consider, as we know that the corner terms originate from the boundary term in the action [33, 57]. Using (2.11) we therefore reach the intriguing conclusion that the late-time bound is

$$
\frac{d \mathcal{C}}{d t} \leq \frac{\alpha}{\pi \hbar} \frac{r_{+}^{2}-r_{-}^{2}}{4 G_{N} \ell^{2}}
$$

for the generalized BTZ black hole. Note that for the pure exotic case $\alpha=0$ the late-time bound vanishes.

We have found, therefore, the first example of a 3-dimensional black hole solution for which the late-time complexity rate growth vanishes. Previous examples have been found only in 2 and 4 dimensions $[34,35]$. In those cases, the inclusion of an additional surface 
term to the action changes the ensemble and consequently the final result for the complexity growth. In general, the calculation of holographic complexity shows how boundary terms that could be at internal boundaries become physically meaningful, and the complexity $=$ action conjecture depends on which action one takes into account. In the case of the exotic black hole, the use of complexity=action seems to suggest the need for introducing an additional proper boundary term (see also the discussion in appendix B) that remains to be explored.

\section{Conclusions}

We have studied the chemistry of generalized exotic BTZ black holes whose thermodynamics are governed by the parameters $\alpha$ and $\gamma$, which serve to swap the roles of mass and angular momentum. The Smarr fomula has been shown to be upheld in all cases. We find that these generalized exotic black holes have a number of interesting features, despite the fact that (as with their $\gamma=0$ BTZ counterparts) they exhibit no interesting phase transition behaviour.

For values of $\alpha>\gamma$, which we term "majority standard" black holes, we show there is no particularly interesting behaviour in the thermodynamic quantities, nor the PV diagrams. However "mostly exotic" black holes, i.e those for which $\gamma>\alpha$, a variety of interesting behaviour is uncovered. In contrast to the mostly regular BTZ black holes, we have found that there exists a maximum volume for sufficiently large values of $J \gg T$. This phenomenon has not been previously seen, and it means that for given values of $(\alpha, \gamma, \Lambda)$ there is a black hole of maximal size. Note that this is not necessarily the same as maximal entropy, since the entropy of these black holes is not proportional to their circumference. We also found that the Gibbs free energy is always greater for exotic black holes than for their BTZ counterparts.

Likewise, we find that the reverse isoperimetric inequality [30] is satisfied for any majority standard black hole. However all sufficiently small majority exotic black holes violate this inequality. The more exotic and smaller the hole, the greater the violation. This suggests a connection between this inequality and the parity properties of the gravitational action, whose meaning remains to be understood. An interesting avenue to explore along these lines is the recently proposed conjecture [58] between the reverse isoperimetric inequality and the negativity of the specific heat at constant volume. Exotic black holes, whose specific heat at constant pressure is always negative (as shown in figure 9) and that always violate the reverse isoperimetric inequality, will provide an interesting test of this conjecture.

Finally a computation of complexity growth for exotic BTZ black holes indicates that in general it is smaller by a factor of $\alpha$ as compared to the standard BTZ black hole, as indicated by (6.10). This parameter encodes the effect of having the gravitational ChernSimons term in the action. Most curiously, the bound on the growth rate vanishes for the purely exotic case $\gamma=1$ and $\alpha=0$. This is because no additional boundary terms are required if $\gamma \neq 0$, and so the computation is the same as the BTZ case. Alternate possibilities are discussed in appendix B. 
Our considerations suggest that a theory of quantum gravity including exotic black holes should exhibit novel properties compared to the usual approaches [27] to the subject. Any such theory will have to account for their distinct thermodynamic behaviour.

\section{Acknowledgments}

We wish to thank Daniel Grumiller, Rodrigo Olea, Olivera Miskovic, Jakob Salzer, Roberto Emparan, Robie Hennigar, Sergey Solodukhin, Hugo Marrochio and Robert C. Myers for interesting discussions and correspondence. This work was supported in part by the Natural Sciences and Engineering Research Council of Canada. The work of AMF was supported by a Swiss Government Excellence Scholarship and is currently supported from ERC Advanced Grant GravBHs692951 and MEC grant FPA2016-76005-C2-2-P.

\section{A BTZ black hole complexity growth}

\section{A.1 General definition of the WdW patch}

The WdW patch is a region with boundaries (see for example the shaded area in the left panel in figure 11). In particular, the WdW patch is defined as the spacetime region of a maximally extended black hole enclosed between four light rays originating from the two chosen equal-times $t_{L}$ and $t_{R}$ on each boundary. Consequently, to evaluate the action in the $\mathrm{WdW}$ patch one needs to include both the standard volume integration and the boundary terms

$$
I=\frac{1}{16 \pi G} \int_{M} \sqrt{-g}(R-2 \Lambda)+\frac{1}{8 \pi G} \int_{\partial M} \sqrt{|h|} K,
$$

where additional corner terms [32] are essential for calculating the rate of change of the action when we shift from the WdW patch at coordinate time $t_{L}$ to the patch at the time $t_{L}+\delta t_{L}$ on the boundary [33]. These "joint contributions" are junction terms where two different boundary surfaces intersect (e.g. see figure 12). Including all these latter contributions, the total action to consider in the calculation is:

$$
I=\frac{1}{16 \pi G}\left[\int_{M}(R-2 \Lambda) \sqrt{-g} d^{4} x+2 \int_{\partial M} K \sqrt{|h|} d \Sigma+2 \sum_{\mathcal{B}_{i}} \operatorname{sign}\left(\mathcal{B}_{i}\right) \oint a_{\mathcal{B}_{i}} d S\right]
$$

where $d \Sigma$ is a volume element in $(D-1)$ and $d S$ is a surface element in $(D-2)$ on the junctions $\mathcal{B}_{i}$ (see figure 11) [32]. For example, in the case of the WdW patch for an uncharged, non-rotating black hole, the difference between the actions evaluated in two different WdW patches, $\mathcal{V}_{1}$ and $\mathcal{V}_{2}$, is given by (see left panel in figure 11)

$$
\delta I_{\mathcal{V}}=I_{\mathcal{V}_{1}}-I_{\mathcal{V}_{2}}-\frac{1}{8 \pi G} \int_{\partial M} K d \Sigma+\frac{1}{8 \pi G}\left[\oint_{\mathcal{B}^{\prime}} a d S-\oint_{\mathcal{B}} a d S\right]
$$

where the only boundary term is evaluated on the surface $\mathcal{S}$ close to the singular spacelike surface [33]. However, in the case of a rotating black hole, there are no space-like boundaries but only null boundaries (see figure 11 right panel) [33]. 

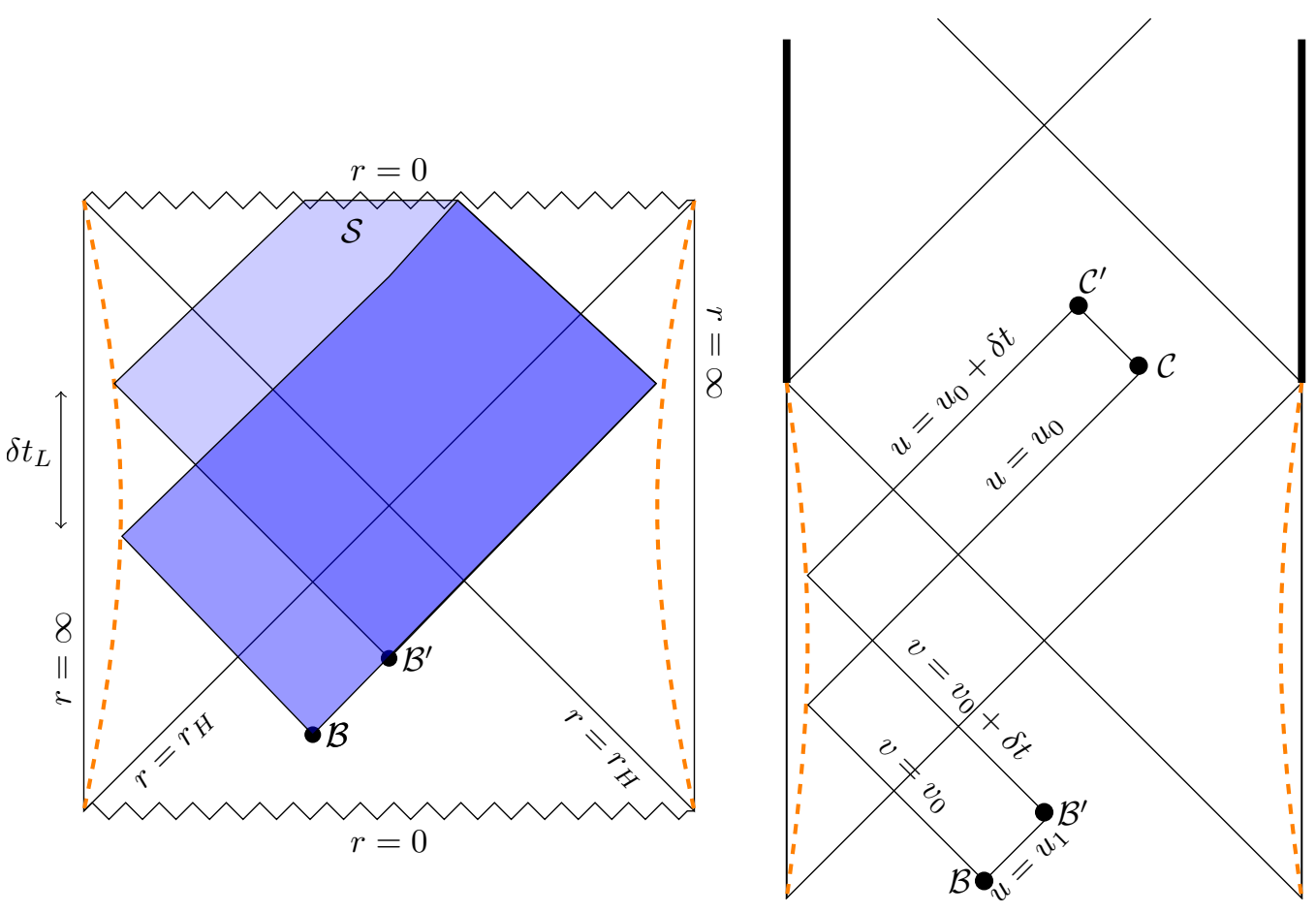

Figure 11. Left: WdW patches for a BTZ black hole in the $J=0$ case. Note that the time on the right boundary is fixed while the time on the left boundary varies. Right: difference between two WdW patches in the case $J \neq 0$.

In general, the null boundaries can be parametrized in such a way that they do not contribute to the action [33].

In the case of a rotating BTZ black hole (as for the charged case) there is no contribution from the boundary term on $\partial M$ (see right panel figure 11) that is indeed behind the inner horizon. So we can remove this term from the variation (A.3) and we are left only with:

$$
\delta I=I_{\mathcal{V}_{1}}-I_{\mathcal{V}_{2}}+\delta I_{\mathcal{B}, \mathcal{B}^{\prime}}+\delta I_{\mathcal{C}, \mathcal{C}^{\prime}}
$$

where we used the notation:

$$
\delta I_{\mathcal{B}, \mathcal{B}^{\prime}}=\frac{1}{8 \pi G}\left[\oint_{\mathcal{B}^{\prime}} a d S-\oint_{\mathcal{B}} a d S\right]
$$

The analysis presented in [33] provides a meaningful comparison of the action between different WdW patches. An extended discussion about possible divergent contributions to the action is presented in [59].

\section{A.2 Rotating BTZ in Einstein-Cartan formalism}

Let us recap the procedure presented for the rotating BTZ black hole. The standard (parity-even) Einstein AdS action in $D=3$ is (now we write also the explicit wedge 
products among forms)

$$
S_{E}=\frac{1}{8 \pi G} \int_{M^{3}}\left[e_{a} \wedge R^{a}-\frac{1}{6 \ell^{2}} \epsilon^{a b c} e_{a} \wedge e_{b} \wedge e_{c}\right]
$$

where instead of working with the metric $g_{\mu \nu}$, we are working with the auxiliary frame fields, or vielbein, $e_{\mu}^{a}$ with $a=0,1,2$ and defined by the relation with the metric

$$
g_{\mu \nu}(x)=e_{\mu}^{a}(x) e_{\nu}^{b}(x) \eta_{a b}
$$

with $\eta_{a b}$ the metric of the flat 3D Minkowski spacetime. The vielbein define a basis in the space of the differential forms. In the "dual notation" (valid only in $D=3$ ) that we used in $S_{E}$, we have

$$
R_{a}=\frac{1}{2} \epsilon_{a b c} R^{b c} \leftrightarrow R^{a b}=-\epsilon^{a b c} R_{c}
$$

therefore, eq. (A.6) can be written:

$$
S_{E}=\frac{1}{16 \pi G} \int_{M^{3}} \epsilon_{a b c}\left(e^{a} \wedge R^{b c}-\frac{1}{3 \ell^{2}} e^{a} \wedge e^{b} \wedge e^{c}\right) .
$$

Since, by definition, one has $e^{a}=e_{\rho}^{a} d x^{\rho}$ and

$$
R^{b c}=\frac{1}{2} R_{\mu \nu}^{b c}(x) d x^{\mu} \wedge d x^{\nu}=\frac{1}{2}\left(e_{\beta}^{b} e_{\gamma}^{c} R_{\mu \nu}^{\beta \gamma}\right) d x^{\mu} \wedge d x^{\nu}
$$

then, eq. (A.9) corresponds to

$$
S_{E}=\frac{1}{16 \pi G} \int_{M^{3}} \epsilon_{a b c}\left[\frac{1}{2}\left(e_{\rho}^{a} e_{\beta}^{b} e_{\gamma}^{c} R_{\mu \nu}^{\beta \gamma}\right)-\frac{1}{3 \ell^{2}} e_{\rho}^{a} e_{\mu}^{b} e_{\nu}^{c}\right] d x^{\rho} \wedge d x^{\mu} \wedge d x^{\nu} .
$$

Using the Levi-Civita in frame components

$$
\epsilon_{\mu \nu \rho}=e^{-1} \epsilon_{a b c} e_{\mu}^{a} e_{\nu}^{b} e_{\rho}^{c}
$$

with $e=\operatorname{det} e_{\mu}^{a}=\sqrt{-g}$, one gets

$$
\begin{aligned}
S_{E} & =\frac{1}{16 \pi G} \int_{M^{3}} e\left(\frac{1}{2} \epsilon_{\rho \beta \gamma} R_{\mu \nu}^{\beta \gamma}-\frac{1}{3 \ell^{2}} \epsilon_{\rho \mu \nu}\right) d x^{\rho} \wedge d x^{\mu} \wedge d x^{\nu} \\
& =\frac{1}{16 \pi G} \int_{M^{3}} d^{3} x \sqrt{-g}\left(\frac{1}{2} \epsilon_{\rho \beta \gamma} \epsilon^{\rho \mu \nu} R_{\mu \nu}^{\beta \gamma}-\frac{1}{3 \ell^{2}} \epsilon_{\rho \mu \nu} \epsilon^{\rho \mu \nu}\right)
\end{aligned}
$$

where in the last line we have used $d x^{\rho} \wedge d x^{\mu} \wedge d x^{\nu}=\epsilon^{\rho \mu \nu} d^{3} x$. Finally, using the following equalities

$$
\begin{aligned}
\frac{1}{2} \epsilon_{\rho \beta \gamma} \epsilon^{\rho \mu \nu} R_{\mu \nu}^{\beta \gamma} & =\frac{1}{2}\left(\delta_{\beta}^{\mu} \delta_{\gamma}^{\nu}-\delta_{\beta}^{\nu} \delta_{\gamma}^{\nu}\right) R_{\mu \nu}^{\beta \gamma}=\frac{1}{2}\left(R_{\mu \nu}^{\mu \nu}-R_{\mu \nu}^{\nu \mu}\right)=R \\
\epsilon_{\rho \mu \nu} \epsilon^{\rho \mu \nu} & =-3 !
\end{aligned}
$$

we get

$$
I_{E}=\frac{1}{16 \pi G} \int_{M^{3}} d^{3} x \sqrt{-g}(R-2 \Lambda)
$$

with negative cosmological constant $\Lambda$

$$
\Lambda=-\frac{(D-2)(D-1)}{2 \ell^{2}}=-1 / \ell^{2}
$$

From this action, we can then proceed in the standard way and get the following contributions to the variation of the action in the WdW patch. 


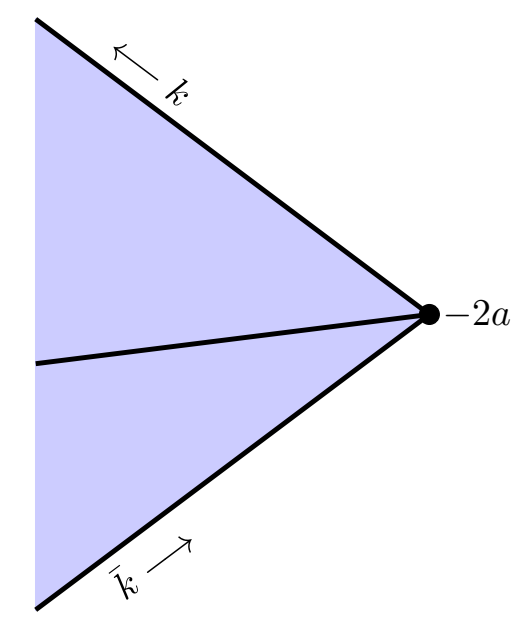

Figure 12. Composition of null/null joints.

\section{A.3 Volume contributions to the WdW patch}

In the presence of a negative cosmological constant, the solution to the equations of motion for the action (2.1) indicates that the Ricci scalar is a constant, $R=6 \Lambda$. Hence the volume contribution to the evolution of the WdW patch is given by

$$
\delta I_{\mathcal{V}}=I_{\mathcal{V}_{1}}-I_{\mathcal{V}_{2}}=-\frac{4}{16 \pi G \ell^{2}} \int \sqrt{-g} d^{3} x
$$

Writing this integral explicitly for the WdW patchs for the rotating black hole in figure 11 (right panel) and considering late times, we obtain

$$
\begin{aligned}
\delta I_{\mathcal{V}} & =-\frac{1}{4 \pi G \ell^{2}} \int_{r_{-}}^{r^{+}} r d r \int_{0}^{2 \pi} d \theta \delta t \\
& =-\left[\left(m-\Omega_{+} j\right)-\left(m-\Omega_{-} j\right)\right] \delta t \\
& =-\frac{r_{+}^{2}-r_{-}^{2}}{4 G \ell^{2}} \delta t
\end{aligned}
$$

with

$$
m=\frac{r_{+}^{2}+r_{-}^{2}}{8 G \ell^{2}}, \quad j=\frac{r_{+} r_{-}}{4 G \ell}, \quad \Omega_{+}=\frac{4 G j}{r_{+}^{2}}, \quad \Omega_{-}=\frac{4 G j}{r_{-}^{2}}
$$

and where we could write the last term as $\delta I_{\mathcal{V}}=-T S \delta t$, since

$$
T=\frac{r_{+}^{2}-r_{-}^{2}}{2 \pi \ell^{2} r_{+}}, \quad S=\frac{\pi r_{+}}{2 G}
$$

While the right-hand side of (A.20) is proportional to the result (6.6), we still need to add the joint (or corner) terms to get the final result. We also note that the rotating BTZ black hole is a special case for which $\left(m-\Omega_{+} j\right)=-\left(m-\Omega_{-} j\right)[31,49]$. 


\section{A.4 Joint contributions}

Now we study the joint terms. It is useful to write

$$
g_{\mu \nu}=\left(\begin{array}{ccc}
r^{2}\left(N^{\phi}\right)^{2}-N^{2} & 0 & r^{2} N^{\phi} \\
0 & N^{-2} & 0 \\
r^{2} N^{\phi} & 0 & r^{2}
\end{array}\right) \quad g^{\mu \nu}=\left(\begin{array}{ccc}
-N^{-2} & 0 & N^{\phi} / N^{2} \\
0 & N^{2} & 0 \\
N^{\phi} / N^{2} & 0 & \frac{1}{r^{2}}-\frac{\left(N^{\phi}\right)^{2}}{N^{2}}
\end{array}\right)
$$

which follow from the rotating BTZ black hole metric (2.3). We want to evaluate the joint terms $\pm 2 \oint_{\mathcal{B}} a d S$ at the intersection between two null-segments, $\mathcal{B}$ and $\mathcal{B}^{\prime}$ that are in this case one dimensional surfaces. The area element in $\mathcal{B}$ and $\mathcal{B}^{\prime}$ is $d S=r d \Omega^{1}=r d \phi$ and

$$
a=\ln \left(-\frac{1}{2} k \cdot \bar{k}\right)
$$

where $k_{a}$ and $\bar{k}_{a}$ are the normals to the null surfaces respectively to $v=v_{0}$ and $u=u_{1}$ that meet in $\mathcal{B}$ (or to $v=v_{0}+\delta t$ in $\mathcal{B}^{\prime}$, see figure 11 ). We can choose the two normals as the affinely parametrized vectors defined by the gradient of the implicit equations of the surfaces [33]:

$$
\begin{aligned}
& k_{a}=-c \partial_{\alpha}\left(v-v_{0}\right)=-c \partial_{\alpha}\left(t-r^{*}\right) \\
& \bar{k}_{a}=\bar{c} \partial_{\alpha}\left(u-u_{1}\right)=\bar{c} \partial_{\alpha}\left(t+r^{*}\right)
\end{aligned}
$$

where $c$ and $\bar{c}$ are normalization constants that define the asymptotic normalization $k \cdot \hat{t}_{L}=-c$ and $\bar{k} \cdot \hat{t}_{R}=-\bar{c}$, where $\hat{t}_{L}=\partial_{\hat{t}_{L}}$ is the asymptotic Killing vector describing the time flow in the left boundary theory and $\hat{t}_{R}=\partial_{t_{R}}$ is the corresponding vector for the right boundary theory, $v_{0}$ and $u_{1}$ are constants and $v:=t-r^{*}, u:=t+r^{*}$. Recalling that $r^{*}=\int g_{11} d r=\int \frac{1}{N^{2}} d r$ we can write the non zero components of the normals:

$$
k_{0}=-c, \quad k_{1}=c N^{-2} \quad \bar{k}_{0}=\bar{c}, \quad \bar{k}_{1}=\bar{c} N^{-2}
$$

so, using this result and the inverse metric, the scalar product in eq. (A.24) is

$$
k \cdot \bar{k}=g^{00} k_{0} \bar{k}_{0}+g^{11} k_{1} \bar{k}_{1}=\frac{2}{N^{2}}(c \bar{c})
$$

so $a=\ln \left(-c \bar{c} / N^{2}\right)$. We can now evaluate the contribution of the joint terms

$$
(16 \pi G) \delta I_{\mathcal{B}, \mathcal{B}^{\prime}}=2\left[\oint_{\mathcal{B}^{\prime}} a d S-\oint_{\mathcal{B}} a d S\right]=\left.2 \int_{0}^{2 \pi} d \phi\left[\ln \left(-\frac{c \bar{c}}{N^{2}}\right) r\right]\right|_{r=r_{\mathcal{B}^{\prime}}} ^{r=r_{\mathcal{B}}}
$$

Since $t=\frac{1}{2}(u+v)$ and $r^{*}=\frac{1}{2}(u-v)$, for $\mathcal{B}$ and $\mathcal{B}^{\prime}$ we have

$$
\begin{aligned}
\mathcal{B} & \equiv\left(v=v_{0}, u=u_{1}\right) \\
\mathcal{B}^{\prime} & \equiv\left(v=v_{0}+\delta t, u=u_{1}\right)
\end{aligned} \Rightarrow \begin{aligned}
r_{\mathcal{B}}^{*} & =\frac{1}{2}\left(u_{1}-v_{0}\right) \\
r_{\mathcal{B}^{\prime}}^{*} & =\frac{1}{2}\left(u_{1}-v_{0}-\delta t\right)
\end{aligned}
$$

and so for small $\delta t$

$$
r_{\mathcal{B}^{\prime}}=r\left(r_{\mathcal{B}^{\prime}}^{*}\right)=r\left(r_{\mathcal{B}}^{*}-\frac{1}{2} \delta t\right) \cong r\left(r_{\mathcal{B}}^{*}\right)-\left.\frac{1}{2} \frac{d r}{d r^{*}}\right|_{r^{*}=r_{\mathcal{B}}^{*}} \delta t=r\left(r_{\mathcal{B}}^{*}\right)-\left.\frac{1}{2} N^{2}\right|_{r^{*}=r_{\mathcal{B}}^{*}} \delta t
$$


Defining $h(r):=r \ln \left(-c \bar{c} N^{-2}\right)$ we have [33]

$$
4 \pi\left[h\left(r_{\mathcal{B}^{\prime}}\right)-h\left(r_{\mathcal{B}}\right)\right]=4 \pi \frac{1}{2}\left[r \frac{d N^{2}}{d r}+N^{2} \ln \left(-\frac{c \bar{c}}{N^{2}}\right)\right] \delta t
$$

where for late times, $r_{\mathcal{B}} \rightarrow r_{+}$and so $N \rightarrow 0$. In this limit, the logarithmic contribution vanishes and the final expression is

$$
\delta I_{\mathcal{B}, \mathcal{B}^{\prime}}=\left.\frac{2 \pi}{16 \pi G}\left[r\left(\frac{2 r}{\ell^{2}}-\frac{32 G^{2} j^{2}}{r^{3}}\right)\right]\right|_{r_{+}} \delta t=\frac{1}{8 G}\left(\frac{2 r_{+}^{2}}{\ell^{2}}-\frac{2 r_{-}^{2}}{\ell^{2}}\right) \delta t
$$

for a rotating BTZ black hole. The same calculation can be done for the junctions $\mathcal{C}$ and $\mathcal{C}^{\prime}$ (see figure 11), yielding

$$
\delta I_{\mathcal{C}, \mathcal{C}^{\prime}}=-\left.\frac{2 \pi}{16 \pi G}\left[r\left(\frac{2 r}{\ell^{2}}-\frac{32 G^{2} j^{2}}{r^{3}}\right)\right]\right|_{r_{-}} \delta t=-\frac{1}{8 G}\left(\frac{2 r_{-}^{2}}{\ell^{2}}-\frac{2 r_{+}^{2}}{\ell^{2}}\right) \delta t
$$

where we used (A.21). Hence

$$
\delta I_{\mathcal{B}, \mathcal{B}^{\prime}}+\delta I_{\mathcal{C}, \mathcal{C}^{\prime}}=\frac{r_{+}^{2}-r_{-}^{2}}{2 G \ell^{2}} \delta t
$$

All together, we find

$$
\delta I_{\mathcal{V}}+\delta I_{\mathcal{B}, \mathcal{B}^{\prime}}+\delta I_{\mathcal{C}, \mathcal{C}^{\prime}}=\frac{\left(r_{+}^{2}-r_{-}^{2}\right)}{4 G \ell^{2}} \delta t
$$

which can also be written as

$$
\begin{aligned}
\delta I_{\mathcal{V}}+\delta I_{\mathcal{B}, \mathcal{B}^{\prime}}+\delta I_{\mathcal{C}, \mathcal{C}^{\prime}} & =2\left[\left(m-\Omega_{+} j\right)-\left(m-\Omega_{-} j\right)\right] \delta t \\
& =2\left[\left(M-\Omega_{+} J\right)-\left(M-\Omega_{-} J\right)\right] \delta t
\end{aligned}
$$

using (2.9) and (2.10) with $\gamma=0$. This is commensurate with (6.6), previously obtained $[31,49]$ via a different method. There is likewise a similar result for a non-rotating BTZ black hole and different regularization procedure [60], and a generalization of this to topological geons has likewise been recently obtained [61].

\section{A.5 Boundary counterterm for rotating BTZ}

Here we calculate the boundary counterterms that are necessary to render the action reparametrization invariant for the null boundaries of the WDW patch. These boundary counterterms play a relevant role in the calculation of complexity growth [62, 63]. For a null hypersurface they in $(2+1)$ dimensions the counterterm contribution to the action is $[33,64]$

$$
I_{\text {boundary }}=\frac{1}{8 \pi G} \int_{\Sigma} d \lambda d r \sqrt{\gamma} \Theta \log (\tilde{L} \Theta)
$$

where $\gamma$ is the induced metric on the null sheet of the WDW patch, $\Theta$ is its expansion given by $\Theta:=\partial_{\lambda} \log \sqrt{\gamma}$ and $\tilde{L}$ is an arbitrary constant. 
Now we calculate the metric $\gamma$ for the rotating BTZ black hole using the procedure illustrated in [65]. Using the rotating-BTZ metric (2.3) we obtain the tortoise coordinate $r_{*}$ that satisfies

$$
g^{\alpha \beta} \partial_{\alpha} v \partial_{\beta} v=g^{t t}+g^{r r}\left(d_{r} r_{\star}(r)\right)^{2}=0
$$

that implies

$$
\left(\frac{d r_{*}}{d r}\right)^{2}=-\frac{g^{t t}}{g^{r r}}=\frac{1}{N^{4}} \rightarrow d r_{*}=\frac{d r}{N^{2}}
$$

transforming the metric $(2.3)$ to

$$
d s^{2}=N^{2}\left(-d t^{2}+d r_{*}^{2}\right)+r^{2}\left(d \phi-N^{\phi} d t\right)^{2}
$$

where $N$ and $N^{\phi}$ are given in (2.4).

Further employing the transformations

$$
d\left(t-r_{*}\right)=d u=-\frac{d U}{\kappa U} \quad d\left(t+r_{*}\right)=d v=\frac{d V}{\kappa V},
$$

yields

$$
d s^{2}=N^{2}\left(\frac{d U}{\kappa U} \frac{d V}{\kappa V}\right)+r^{2}\left[d \phi-\frac{N^{\phi}}{2 \kappa}\left(-\frac{d U}{U}+\frac{d V}{V}\right)\right]^{2}
$$

from (A.39).

Although the first term in (A.41) is regular at the horizon, the 2nd term is not regular at either of $U=0$ or $V=0$. This problem can be dealt with by defining $[65,66]$

$$
\varphi_{ \pm}=\phi \pm \int \frac{N^{\phi}}{N^{2}} d r
$$

yielding for (A.41)

$$
d s^{2}=N^{2}\left(\frac{d U}{\kappa U} \frac{d V}{\kappa V}\right)+r^{2}\left[d \varphi_{+}-N^{\phi} d v\right]^{2}
$$

valid at $U=0$ and

$$
d s^{2}=N^{2}\left(\frac{d U}{\kappa U} \frac{d V}{\kappa V}\right)+r^{2}\left[d \varphi_{-}-N^{\phi} d u\right]^{2}
$$

valid at $V=0$. The induced metric is given

$$
\begin{aligned}
& d x^{A} d x^{B} \gamma_{A B}=d s^{2}(U=\text { const })=r^{2}\left[d \varphi_{+}-N^{\phi} d v\right]^{2} \\
& d x^{A} d x^{B} \gamma_{A B}=d s^{2}(V=\text { const })=r^{2}\left[d \varphi_{-}-N^{\phi} d u\right]^{2}
\end{aligned}
$$

and so the determinant is $\gamma=r^{2}$.

Using the affine parameter $\lambda=r / \alpha$, the expansion parameter $\Theta$ is

$$
\Theta=\partial_{\lambda} \log (\sqrt{\gamma})=\frac{\alpha}{r}
$$


which is the same counterterm as that for the non-rotating-BTZ black hole. Hence

$$
\begin{aligned}
\Delta I_{\Sigma}^{\mathrm{BTZ}} & =\frac{\Omega_{k, 1}}{4 \pi G} \int_{0}^{r_{\max }} d r \log \left(\frac{\alpha \tilde{L}}{r}\right)+\frac{\Omega_{k, 1}}{4 \pi G} \int_{r_{\mathrm{m}}}^{r_{\max }} d r \log \left(\frac{\alpha \tilde{L}}{r}\right) \\
& =\frac{1}{G} r_{\max }\left(\log \frac{r_{\max }}{\alpha \tilde{L}}-1\right)+\frac{1}{2 G} r_{\mathrm{m}}\left(\log \frac{r_{\mathrm{m}}}{\alpha \tilde{L}}-1\right)
\end{aligned}
$$

considering that there are two past null boundaries and two future null boundaries in total.

The quantity $r_{\max }$ is the position of the UV regulator surface (at the boundary), and $r_{m}$ is the location of the intersection of the two past null boundaries; we shall set $r_{m}=0$ when these boundaries end on the past singularity.

However the causal structure of the rotating BTZ black hole is similar to the charged AdS black hole: there are inner and outer horizons, and the WDW patch does not end on the singularity. Specifically $r_{\mathrm{m}}$ never ends on the singularity (see right panel in figure 11); rather the intersection points are at $r(\mathcal{B}), r\left(\mathcal{B}^{\prime}\right), r(\mathcal{C}), r\left(\mathcal{C}^{\prime}\right)$.

Since the counterterm for the $\mathrm{AdS}_{3}$ is

$$
\Delta I_{\Sigma}^{\mathrm{AdS}}=-\frac{1}{2 G} r_{\max }\left(\log \frac{r_{\max }}{\alpha \tilde{L}}-1\right)
$$

we obtain from (A.48) the total regularized counterterm

$$
\Delta I^{\mathrm{reg}}=\Delta I_{\Sigma}^{\mathrm{BTZ}}-2 \Delta I_{\Sigma}^{\mathrm{AdS}}=\frac{1}{2 G} r_{\mathrm{m}}\left(\log \frac{r_{\mathrm{m}}}{\alpha \tilde{L}}-1\right) .
$$

We can show that adding this term to the total action cancels the dependence on $\alpha$ (i.e. is independent of the normalization constant $\alpha$ appearing in the null normals). If we adopt for this subsection the normalization of the null vectors in (A.25) to be the same:

$$
k \cdot \hat{t}_{L}=\bar{k} \cdot \hat{t}_{R}= \pm \alpha
$$

where the sign + is for the future null surfaces and the sign - is for the past null surfaces, the join contribution (A.27) is

$$
I_{\mathrm{jnt}}=\frac{1}{8 \pi G} \int_{0}^{2 \pi} d \phi r_{\mathrm{m}} \log \left(\frac{\alpha^{2}}{N^{2}\left(r_{m}\right)}\right)=\frac{1}{4 G} r_{\mathrm{m}} \log \left(\frac{\alpha^{2}}{N^{2}\left(r_{m}\right)}\right)
$$

therefore, if we sum

$$
\begin{aligned}
\Delta I^{\mathrm{reg}}+I_{\mathrm{jnt}} & =\frac{r_{m}}{2 G}\left[\log \frac{r_{\mathrm{m}}}{\alpha \tilde{L}}-1+\frac{1}{2} \log \frac{\alpha^{2}}{N^{2}\left(r_{\mathrm{m}}\right)}\right] \\
& =\frac{r_{\mathrm{m}}}{2 G}\left[\log \frac{r_{\mathrm{m}}}{\alpha \tilde{L}}+\log \frac{\alpha}{N\left(r_{\mathrm{m}}\right)}-1\right] \\
& =\frac{r_{\mathrm{m}}}{2 G}\left[\log \frac{r_{\mathrm{m}}}{\tilde{L} N\left(r_{\mathrm{m}}\right)}-1\right]
\end{aligned}
$$

we find that the result is independent of the normalization constant present in the null normal normalization. 


\section{B Alternate possibilities}

The preceding calculation assumed that the boundary term in the action (2.11) was a functional of the boundary metric and unit normal only. Using the tetrad formalism, it is in fact possible to see that the variation of the various terms in the action (2.11) with respect to $e_{a}$ and $\omega_{a}$ give the following boundary terms:

$$
\begin{aligned}
\delta_{\omega} I_{\mathrm{EH}} & =\int d\left(e_{a} \wedge \delta \omega^{a}\right) \\
\delta_{e} I_{\mathrm{GCS}} & =\int d\left(e_{a} \wedge \delta e^{a}\right) \\
\delta_{\omega} I_{\mathrm{GCS}} & =\int d\left(\omega_{a} \wedge \delta \omega^{a}\right)
\end{aligned}
$$

and while eq. (B.1) gives the well-known GHY term and the second (B.2) can be canceled using the boundary condition for $e^{a}$, a boundary term that could cancel (B.3) could play a role in the calculation of the complexity growth. However no such boundary term exists. Furthermore, a Fefferman-Graham coordinate expansion indicates that it depends only on variations of the leading part of the asymptotic metric [46], and so vanishes in spacetimes having asymptotically AdS boundary conditions. Note also that since the quantity $\sqrt{|h|} K-2 \sqrt{|h|} / \ell$ is a topological invariant of the background metric in $(2+1)$ dimensions $[37,43,56]$, there is some ambiguity in choosing the boundary term in (2.11); this does not affect the computations in the previous appendix.

Another hint that something interesting can happen in this case is if one wants to analyze the connection between the thermodynamic volume and the complexity growth given in [50]. It has been proposed indeed that the complexification rate could be proportional to the product $P V$ (this is called "Complexity=Volume" 2.0). This is because, the complexity growth should be proportional to the number of degrees of freedom that are in the boundary CFT given by the central charge multiplied for the number of lattice sites $n$ (roughly the volume divided by the lattice spacing) multiplied by the number of circuit steps $n_{c}$.

In $D$ spacetime dimensions (setting $G_{N}=1$ ), the central charge scales as $c \sim \ell^{D-2} \sim$ $P^{-(D-2) / 2}$, and the number of sites $n \sim V / \epsilon^{D-1}$, where the lattice spacing $\epsilon \sim \ell \sim \sqrt{\frac{1}{P}}$. The number of circuit steps $n_{c} \sim \Delta t / \epsilon$, so

$$
\mathcal{C} \propto \frac{1}{P^{(D-2) / 2}} \times V P^{(D-1) / 2} \times \Delta t \sqrt{P}=P V \Delta t
$$

up to some normalization. ${ }^{1}$ In the standard bulk BTZ case the Brown-Henneaux formula

$$
c=\frac{3 \ell}{2 G_{N}} \propto \sqrt{\frac{1}{P}}
$$

suggests that one can write [50]

$$
\mathcal{C}=P V\left(t_{L}+t_{R}\right)
$$

\footnotetext{
${ }^{1}$ We are grateful to Robie Hennigar for clarification of this argument.
} 
for $D=3$. However, if we add the GCS term to the action, it has been shown that the boundary CFT has different values of the central charge [29, 39]

$$
c_{L}=\frac{3}{2} \frac{(\alpha-\gamma) \ell}{G_{N}}, \quad c_{R}=\frac{3}{2} \frac{(\alpha+\gamma) \ell}{G_{N}} .
$$

Now, in order to obtain the well known result for the BTZ black hole, we need to define the quantity $c_{+}=\left(c_{L}+c_{R}\right) / 2$. This quantity has been derived in [39] using the conformal anomaly to determine the covariantly conserved stress-energy tensor in the presence of the gravitational Chern-Simons term in the action. Using $c_{+}$, we obtain the correct result both in the standard case $(\gamma=0)$, when $c_{L}=c_{R}$ and the quantity $c_{+}$is equal to (B.5) giving the result (B.6), and for the more general exotic case, our final result eq. (6.10).

Open Access. This article is distributed under the terms of the Creative Commons Attribution License (CC-BY 4.0), which permits any use, distribution and reproduction in any medium, provided the original author(s) and source are credited.

\section{References}

[1] J.D. Bekenstein, Black holes and entropy, Phys. Rev. D 7 (1973) 2333 [inSPIRE].

[2] S.W. Hawking, Black holes in general relativity, Commun. Math. Phys. 25 (1972) 152 [INSPIRE].

[3] J.M. Bardeen, B. Carter and S.W. Hawking, The Four laws of black hole mechanics, Commun. Math. Phys. 31 (1973) 161 [INSPIRE].

[4] J.M. Maldacena, The Large $N$ limit of superconformal field theories and supergravity, Int. J. Theor. Phys. 38 (1999) 1113 [hep-th/9711200] [INSPIRE].

[5] D. Kubiznak and R.B. Mann, Black hole chemistry, Can. J. Phys. 93 (2015) 999 [arXiv: 1404.2126] [INSPIRE].

[6] D. Kastor, S. Ray and J. Traschen, Enthalpy and the Mechanics of AdS Black Holes, Class. Quant. Grav. 26 (2009) 195011 [arXiv: 0904.2765] [INSPIRE].

[7] B.P. Dolan, The cosmological constant and the black hole equation of state, Class. Quant. Grav. 28 (2011) 125020 [arXiv: 1008.5023] [INSPIRE].

[8] B.P. Dolan, Pressure and volume in the first law of black hole thermodynamics, Class. Quant. Grav. 28 (2011) 235017 [arXiv:1106.6260] [INSPIRE].

[9] B.P. Dolan, Compressibility of rotating black holes, Phys. Rev. D 84 (2011) 127503 [arXiv: 1109.0198] [INSPIRE].

[10] D. Kubiznak and R.B. Mann, P-V criticality of charged AdS black holes, JHEP 07 (2012) 033 [arXiv: 1205.0559] [INSPIRE].

[11] N. Altamirano, D. Kubiznak and R.B. Mann, Reentrant phase transitions in rotating anti-de Sitter black holes, Phys. Rev. D 88 (2013) 101502 [arXiv:1306.5756] [INSPIRE].

[12] S. Gunasekaran, R.B. Mann and D. Kubiznak, Extended phase space thermodynamics for charged and rotating black holes and Born-Infeld vacuum polarization, JHEP 11 (2012) 110 [arXiv: 1208.6251] [INSPIRE]. 
[13] A.M. Frassino, D. Kubiznak, R.B. Mann and F. Simovic, Multiple Reentrant Phase Transitions and Triple Points in Lovelock Thermodynamics, JHEP 09 (2014) 080 [arXiv: 1406.7015] [INSPIRE].

[14] N. Altamirano, D. Kubizñák, R.B. Mann and Z. Sherkatghanad, Kerr-AdS analogue of triple point and solid/liquid/gas phase transition, Class. Quant. Grav. 31 (2014) 042001 [arXiv: 1308.2672] [INSPIRE].

[15] A.M. Frassino, M. Bleicher and R.B. Mann, Unruh thermal hadronization and the cosmological constant, Phys. Lett. B $\mathbf{7 8 0}$ (2018) 93 [arXiv:1712.09372] [INSPIRE].

[16] A. Karch and B. Robinson, Holographic Black Hole Chemistry, JHEP 12 (2015) 073 [arXiv: 1510.02472] [INSPIRE].

[17] M. Sinamuli and R.B. Mann, Super-Entropic Black Holes and the Kerr-CFT Correspondence, JHEP 08 (2016) 148 [arXiv:1512.07597] [inSPIRE].

[18] M. Sinamuli and R.B. Mann, Higher Order Corrections to Holographic Black Hole Chemistry, Phys. Rev. D 96 (2017) 086008 [arXiv:1706.04259] [INSPIRE].

[19] C.V. Johnson, Holographic Heat Engines, Class. Quant. Grav. 31 (2014) 205002 [arXiv: 1404.5982] [INSPIRE].

[20] E. Caceres, P.H. Nguyen and J.F. Pedraza, Holographic entanglement entropy and the extended phase structure of STU black holes, JHEP 09 (2015) 184 [arXiv:1507.06069] [INSPIRE].

[21] P.H. Nguyen, An equal area law for holographic entanglement entropy of the AdS-RN black hole, JHEP 12 (2015) 139 [arXiv:1508.01955] [INSPIRE].

[22] R.A. Hennigar, F. McCarthy, A. Ballon and R.B. Mann, Holographic heat engines: general considerations and rotating black holes, Class. Quant. Grav. 34 (2017) 175005 [arXiv: 1704.02314] [INSPIRE].

[23] R.A. Hennigar, R.B. Mann and E. Tjoa, Superfluid Black Holes, Phys. Rev. Lett. 118 (2017) 021301 [arXiv: 1609.02564] [INSPIRE].

[24] D. Kubiznak, R.B. Mann and M. Teo, Black hole chemistry: thermodynamics with Lambda, Class. Quant. Grav. 34 (2017) 063001 [arXiv:1608.06147] [INSPIRE].

[25] L. Smarr, Mass formula for Kerr black holes, Phys. Rev. Lett. 30 (1973) 71 [Erratum ibid. 30 (1973) 521] [INSPIRE].

[26] A.M. Frassino, R.B. Mann and J.R. Mureika, Lower-Dimensional Black Hole Chemistry, Phys. Rev. D 92 (2015) 124069 [arXiv:1509.05481] [INSPIRE].

[27] S. Carlip, J. Gegenberg and R.B. Mann, Black holes in three-dimensional topological gravity, Phys. Rev. D 51 (1995) 6854 [gr-qc/9410021] [INSPIRE].

[28] M. Bañados, A. Gomberoff and C. Martinez, Anti-de Sitter space and black holes, Class. Quant. Grav. 15 (1998) 3575 [hep-th/9805087] [INSPIRE].

[29] P.K. Townsend and B. Zhang, Thermodynamics of "Exotic" Bañados-Teitelboim-Zanelli Black Holes, Phys. Rev. Lett. 110 (2013) 241302 [arXiv:1302.3874] [inSPIRE].

[30] M. Cvetič, G.W. Gibbons, D. Kubiznak and C.N. Pope, Black Hole Enthalpy and an Entropy Inequality for the Thermodynamic Volume, Phys. Rev. D 84 (2011) 024037 [arXiv: 1012.2888] [INSPIRE]. 
[31] R.-G. Cai, S.-M. Ruan, S.-J. Wang, R.-Q. Yang and R.-H. Peng, Action growth for AdS black holes, JHEP 09 (2016) 161 [arXiv:1606.08307] [INSPIRE].

[32] I.S. Booth, Metric based Hamiltonians, null boundaries and isolated horizons, Class. Quant. Grav. 18 (2001) 4239 [gr-qc/0105009] [INSPIRE].

[33] L. Lehner, R.C. Myers, E. Poisson and R.D. Sorkin, Gravitational action with null boundaries, Phys. Rev. D 94 (2016) 084046 [arXiv: 1609.00207] [INSPIRE].

[34] A.R. Brown, H. Gharibyan, H.W. Lin, L. Susskind, L. Thorlacius and Y. Zhao, Complexity of Jackiw-Teitelboim gravity, Phys. Rev. D 99 (2019) 046016 [arXiv:1810.08741] [INSPIRE].

[35] K. Goto, H. Marrochio, R.C. Myers, L. Queimada and B. Yoshida, Holographic Complexity Equals Which Action?, JHEP 02 (2019) 160 [arXiv:1901.00014] [INSPIRE].

[36] E. Witten, (2+1)-Dimensional Gravity as an Exactly Soluble System, Nucl. Phys. B 311 (1988) 46 [INSPIRE].

[37] O. Mišković and R. Olea, Background-independent charges in Topologically Massive Gravity, JHEP 12 (2009) 046 [arXiv:0909.2275] [INSPIRE].

[38] B. Zhang, The mass formula for an exotic BTZ black hole, Annals Phys. 367 (2016) 280 [arXiv: 1603.08600] [INSPIRE].

[39] S.N. Solodukhin, Holography with gravitational Chern-Simons, Phys. Rev. D 74 (2006) 024015 [hep-th/0509148] [INSPIRE].

[40] G. Immirzi, Real and complex connections for canonical gravity, Class. Quant. Grav. 14 (1997) L177 [gr-qc/9612030] [inSPIRE].

[41] C. Meusburger and B.J. Schroers, Generalised Chern-Simons actions for $3 d$ gravity and kappa-Poincaré symmetry, Nucl. Phys. B 806 (2009) 462 [arXiv:0805.3318] [InSPIRE].

[42] V. Bonzom and E.R. Livine, A Immirzi-like parameter for $3 d$ quantum gravity, Class. Quant. Grav. 25 (2008) 195024 [arXiv: 0801.4241] [INSPIRE].

[43] O. Mišković and R. Olea, On boundary conditions in three-dimensional AdS gravity, Phys. Lett. B 640 (2006) 101 [hep-th/0603092] [INSPIRE].

[44] M. Bañados and F. Mendez, A Note on covariant action integrals in three-dimensions, Phys. Rev. D 58 (1998) 104014 [hep-th/9806065] [INSPIRE].

[45] E. Witten, Anti-de Sitter space, thermal phase transition and confinement in gauge theories, Adv. Theor. Math. Phys. 2 (1998) 505 [hep-th/9803131] [INSPIRE].

[46] P. Kraus and F. Larsen, Holographic gravitational anomalies, JHEP 01 (2006) 022 [hep-th/0508218] [INSPIRE].

[47] C. Liang, L. Gong and B. Zhang, Smarr formula for BTZ black holes in general three-dimensional gravity models, Class. Quant. Grav. 34 (2017) 035017 [arXiv:1701.03223] [INSPIRE].

[48] C. Erices, O. Fuentealba and M. Riquelme, Electrically charged black hole on AdS $S_{3}$ : Scale invariance and the Smarr formula, Phys. Rev. D 97 (2018) 024037 [arXiv:1710.05962] [INSPIRE].

[49] A.R. Brown, D.A. Roberts, L. Susskind, B. Swingle and Y. Zhao, Complexity, action and black holes, Phys. Rev. D 93 (2016) 086006 [arXiv: 1512.04993] [INSPIRE]. 
[50] J. Couch, W. Fischler and P.H. Nguyen, Noether charge, black hole volume and complexity, JHEP 03 (2017) 119 [arXiv: 1610.02038] [INSPIRE].

[51] J. Couch, S. Eccles, T. Jacobson and P. Nguyen, Holographic Complexity and Volume, JHEP 11 (2018) 044 [arXiv: 1807.02186] [INSPIRE].

[52] D. Grumiller and M. Riegler, Most general AdS $S_{3}$ boundary conditions, JHEP 10 (2016) 023 [arXiv: 1608.01308] [INSPIRE].

[53] D. Grumiller, R.B. Mann and R. McNees, Dirichlet boundary value problem for Chern-Simons modified gravity, Phys. Rev. D 78 (2008) 081502 [arXiv:0803.1485] [INSPIRE].

[54] G. Arcioni, M. Blau and M. O'Loughlin, On the boundary dynamics of Chern-Simons gravity, JHEP 01 (2003) 067 [hep-th/0210089] [INSPIRE].

[55] M.-I. Park, BTZ black hole with gravitational Chern-Simons: Thermodynamics and statistical entropy, Phys. Rev. D 77 (2008) 026011 [hep-th/0608165] [INSPIRE].

[56] S. Detournay, D. Grumiller, F. Schöller and J. Simón, Variational principle and one-point functions in three-dimensional flat space Einstein gravity, Phys. Rev. D 89 (2014) 084061 [arXiv: 1402.3687] [INSPIRE].

[57] I. Jubb, J. Samuel, R. Sorkin and S. Surya, Boundary and Corner Terms in the Action for General Relativity, Class. Quant. Grav. 34 (2017) 065006 [arXiv: 1612.00149] [INSPIRE].

[58] C.V. Johnson, Instability of Super-Entropic Black Holes in Extended Thermodynamics, arXiv: 1906.00993 [INSPIRE].

[59] D. Carmi, R.C. Myers and P. Rath, Comments on Holographic Complexity, JHEP 03 (2017) 118 [arXiv: 1612.00433] [INSPIRE].

[60] D. Carmi, S. Chapman, H. Marrochio, R.C. Myers and S. Sugishita, On the Time Dependence of Holographic Complexity, JHEP 11 (2017) 188 [arXiv:1709.10184] [INSPIRE].

[61] M. Sinamuli and R.B. Mann, Topological and time dependence of the action-complexity relation, Phys. Rev. D 98 (2018) 026005 [arXiv:1804.07333] [INSPIRE].

[62] C.A. Agón, M. Headrick and B. Swingle, Subsystem Complexity and Holography, JHEP 02 (2019) 145 [arXiv: 1804.01561] [InSPIRE].

[63] E. Caceres and M.-L. Xiao, Complexity-action of subregions with corners, JHEP 03 (2019) 062 [arXiv: 1809.09356] [INSPIRE].

[64] A. Reynolds and S.F. Ross, Divergences in Holographic Complexity, Class. Quant. Grav. 34 (2017) 105004 [arXiv: 1612.05439] [INSPIRE].

[65] A.A. Balushi and R.B. Mann, Null hypersurfaces in Kerr-(A)dS spacetimes, arXiv: 1909.06419 [INSPIRE].

[66] J.S.F. Chan, K.C.K. Chan and R.B. Mann, Interior structure of a charged spinning black hole in (2+1)-dimensions, Phys. Rev. D 54 (1996) 1535 [gr-qc/9406049] [InSPIRE]. 